\title{
Combinatorics and topology related to involutions in Coxeter groups
}

\section{Mikael Hansson}

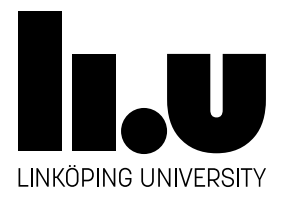

Department of Mathematics

Linköping University

SE-581 83 Linköping, Sweden

Linköping 2018 
Combinatorics and topology related to involutions in Coxeter groups Mikael Hansson

Department of Mathematics

Linköping University

SE-581 83 Linköping, Sweden

mikael.hansson@liu.se

Linköping Studies in Science and Technology, Dissertation No. 1924

ISSN 0345-7524

ISBN 978-91-7685-334-4

Copyright (c) 2018 Mikael Hansson, unless otherwise noted

Printed by LiU-Tryck, Linköping, Sweden, 2018 


\section{Abstract}

This dissertation consists of three papers in combinatorial Coxeter group theory.

A Coxeter group is a group $W$ generated by a set $S$, where all relations can be derived from the relations $s^{2}=e$ for all $s \in S$, and $\left(s s^{\prime}\right)^{m\left(s, s^{\prime}\right)}=e$ for some pairs of generators $s \neq s^{\prime}$ in $S$, where $e \in W$ is the identity element and $m\left(s, s^{\prime}\right)$ is an integer satisfying that $m\left(s, s^{\prime}\right)=m\left(s^{\prime}, s\right) \geq 2$. Two prominent examples of Coxeter groups are provided by the symmetric group $S_{n}$ (i.e., the set of permutations of $\{1,2, \ldots, n\}$ ) and finite reflection groups (i.e., finite groups generated by reflections in some real euclidean space). There are also important infinite Coxeter groups, e.g., affine reflection groups.

Every Coxeter group can be equipped with various natural partial orders, the most important of which is the Bruhat order. Any subset of a Coxeter group can then be viewed as an induced subposet.

In Paper A, we study certain posets of this kind, namely, unions of conjugacy classes of involutions in the symmetric group. We obtain a complete classification of the posets that are pure (i.e., all maximal chains have the same length). In particular, we prove that the set of involutions with exactly one fixed point is pure, which settles a conjecture of Hultman in the affirmative. When the posets are pure, we give their rank functions. We also give a short, new proof of the EL-shellability of the set of fixed-point-free involutions, established by Can, Cherniavsky, and Twelbeck.

Paper B also deals with involutions in Coxeter groups. Given an involutive automorphism $\theta$ of a Coxeter system $(W, S)$, let

$$
\mathfrak{I}(\theta)=\left\{w \in W \mid \theta(w)=w^{-1}\right\}
$$

be the set of twisted involutions. In particular, $\mathfrak{I}(\mathrm{id})$ is the set of ordinary involutions in $W$. It is known that twisted involutions can be represented by words in the alphabet $\underline{S}=\{\underline{s} \mid s \in S\}$, called $\underline{S}$-expressions. If $s s^{\prime}$ has finite order $m\left(s, s^{\prime}\right)$, let a braid move be the replacement of $\underline{s} \underline{s}^{\prime} \underline{\underline{s}} \cdots$ by $\underline{s}^{\prime} \underline{s} \underline{s}^{\prime} \cdots$, both consisting of $m\left(s, s^{\prime}\right)$ letters. We prove a word property for $\mathfrak{I}(\theta)$, for 
any Coxeter system $(W, S)$ with any $\theta$. More precisely, we provide a minimal set of moves, easily determined from the Coxeter graph of $(W, S)$, that can be added to the braid moves in order to connect all reduced $\underline{S}$-expressions for any given $w \in \mathfrak{I}(\theta)$. This improves upon a result of Hamaker, Marberg, and Pawlowski, and generalises similar statements valid in certain types due to $\mathrm{Hu}$, Zhang, $\mathrm{Wu}$, and Marberg.

In Paper $\mathrm{C}$, we investigate the topology of (the order complexes of) certain posets, called pircons. A special partial matching (SPM) on a poset is a matching of the Hasse diagram satisfying certain extra conditions. An SPM without fixed points is precisely a special matching as defined by Brenti. Let a pircon be a poset in which every non-trivial principal order ideal is finite and admits an SPM. Thus pircons generalise Marietti's zircons. Our main result is that every open interval in a pircon is a PL ball or a PL sphere.

An important subset of $\mathfrak{I}(\theta)$ is the set $\iota(\theta)=\left\{\theta\left(w^{-1}\right) w \mid w \in W\right\}$ of twisted identities. We prove that if $\theta$ does not flip any edges with odd labels in the Coxeter graph, then $\iota(\theta)$, with the order induced by the Bruhat order on $W$, is a pircon. Hence, its open intervals are PL balls or spheres, which confirms a conjecture of Hultman. It is also demonstrated that Bruhat orders on Rains and Vazirani's quasiparabolic $W$-sets (under a boundedness assumption) form pircons. In particular, this applies to all parabolic quotients of Coxeter groups. 


\section{Populärvetenskaplig sammanfattning}

Den här avhandlingen består av tre artiklar inom kombinatorisk Coxetergruppteori.

Coxetergrupper är viktiga inom algebra, geometri och kombinatorik. Två centrala Coxetergrupper är den symmetriska gruppen $S_{n}$ och ändliga reflektionsgrupper. Den förra består av alla permutationer av talen $1,2, \ldots, n$, d.v.s. alla uppräkningar av talen, till exempel 4,2,5,1,3 eller 5,3,4,1,2 om $n=5$. Ett exempel på en ändlig reflektionsgrupp är gruppen som genereras av reflektionerna av en kvadrat i dess fyra symmetrilinjer. Om man exempelvis speglar den första kvadraten nedan i den vågräta symmetrilinjen fås den andra kvadraten. Speglar man sedan den andra kvadraten i den lodräta symmetrilinjen fås den tredje kvadraten.
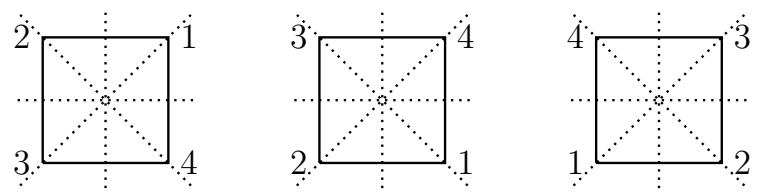

Det finns också viktiga Coxetergrupper med oändligt många element.

En partialordnad mängd (eller pomängd) är en mängd där en del element är större än andra, men det finns i allmänhet också element som inte är jämförbara med varandra (inget av elementen är större än det andra). Varje Coxetergrupp $W$ kan partialordnas på olika naturliga sätt. På så vis blir $W$ en pomängd. Den viktigaste partialordningen på en Coxetergrupp är den så kallade bruhatordningen.

En involution i $S_{n}$ är en permutation som fås genom att bara talen inom vissa par byter plats med varandra. Till exempel är 4,2,5, 1,3 en involution (1 byter plats med 4 och 3 byter plats med 5), men 5,3,4,1,2 är det inte. Det är naturligt att betrakta mängden $I_{n}$ av alla involutioner som en pomängd, där ordningen bestäms av bruhatordningen på $S_{n}$. Man kan också betrakta 
vissa delmängder av $I_{n}$ som pomängder, till exempel $F_{n}^{1}$, de involutioner som har exakt en fixpunkt. I involutionen 4,2,5,1,3 är 2 den enda fixpunkten, ty talet 2 står på plats 2 .

I den första artikeln klassificerar vi de pomängder som är rena. Det är en viktig egenskap som en pomängd kan ha, och medför att elementen i pomängden kan delas in i olika "nivåer". Vi bestämmer också rangfunktionen av de rena pomängderna. Den beskriver vilken nivå varje element tillhör. Vi ger också ett kort, nytt bevis av att $F_{n}^{0}$, de involutioner som inte har några fixpunkter, är EL-skalbar. Det betyder att pomängden kan "märkas" på ett sätt som säger något om dess topologi.

Den utmärkande egenskapen hos $I_{n}$ är att om man utför samma involution två gånger så får man $1,2, \ldots, n$, till exempel om 3 byter plats med 5 två gånger. På samma sätt är ett element i en godtycklig Coxetergrupp en involution om samma element en gång till "tar oss tillbaka till där vi började". Till exempel är spegling i exakt en symmetrilinje i figuren på förra sidan en involution. Involutioner kan generaliseras till vrickade involutioner. Allt som påstås om involutioner i nästa stycke gäller även för vrickade involutioner.

Involutioner kan representeras av ord med bokstäver från ett alfabet som bestäms av Coxetergruppen. Varje involution kan i allmänhet representeras av flera olika ord, även om man kräver att orden ska vara reducerade, d.v.s. så korta som möjligt. Ett drag är ett sätt att förändra ett reducerat ord så att det fortfarande representerar samma involution. I den andra artikeln tar vi fram ett antal drag sådana att om man har ett reducerat ord för en godtycklig involution, så kan man producera alla reducerade ord för denna involution med hjälp av dessa drag. Tack vara detta resultat kan man alltid avgöra huruvida två ord representerar samma involution.

Zirkoner är en välstuderad typ av pomängder. Till exempel är mängden av alla vrickade involutioner en zirkon. I den tredje artikeln beskriver vi strukturen hos så kallade pirkoner med hjälp av begrepp från topologi. Alla zirkoner är pirkoner, men det finns också pirkoner som inte är zirkoner.

Ett öppet intervall $(x, y)$ i en pomängd består av alla element som är större än $x$ och mindre än $y$. Vårt huvudresultat är att varje öppet intervall i en pirkon har topologin av ett klot eller en sfär. Det betyder att intervallen "ser ut som" klot eller sfärer, eller deras generaliseringar i högre eller lägre dimensioner. Vidare visar vi att vissa välkända pomängder utgör pirkoner. Det motiverar varför pirkoner är intressanta att studera. Till exempel är mängden av alla vrickade identiteter, som är en viktig delmängd av mängden av alla vrickade involutioner, ofta en pirkon. 


\section{Acknowledgements}

First of all, I would like to thank my supervisor, Axel Hultman. Thank you for many interesting discussions, thoughtful comments, and your patience and support. I also want to thank my second supervisor, Jan Snellman, and my master's thesis advisor, Jörgen Backelin, who introduced me to mathematical research. A big thank you goes to my co-author Nancy Abdallah. Many thanks go to all my colleagues and fellow PhD students, past and present, at the Department of Mathematics for making it such a pleasant workplace. Among them, in particular, I want to thank Arpan Ghosh, who has been a great friend for the past four years. Finally, I want to thank my family for always believing in me. 



\section{List of included papers}

The dissertation is based on the following three papers:

[A] M. Hansson, The Bruhat order on conjugation-invariant sets of involutions in the symmetric group, Journal of Algebraic Combinatorics 44 (2016), 849-862.

[B] M. Hansson and A. Hultman, A word property for twisted involutions in Coxeter groups (2017), preprint submitted for publication.

[C] N. Abdallah, M. Hansson, and A. Hultman, Topology of posets with special partial matchings (2017), preprint submitted for publication.

In Papers $\mathrm{B}$ and $\mathrm{C}$, the ideas were developed and the results derived jointly by the authors. I led the writing of both papers.

Extended abstracts of Papers A and B have been presented by me at the following conferences:

- FPSAC 2015 in Daejeon, South Korea

- EuroComb 2015 in Bergen, Norway

- EuroComb 2017 in Vienna, Austria

An extended abstract based on Paper $\mathrm{C}$ has been accepted for presentation at FPSAC 2018 in Hanover, New Hampshire, USA.

The following article, partly based on my master's thesis, was also written during my doctoral studies, but is not part of the dissertation:

- M. Hansson, Generalised Ramsey numbers for two sets of cycles, Discrete Applied Mathematics 238 (2018), 86-94.

\footnotetext{
${ }^{1}$ International Conference on Formal Power Series and Algebraic Combinatorics

${ }^{2}$ European Conference on Combinatorics, Graph Theory and Applications
} 



\section{Contents}

I Introduction 1

1 Posets 3

2 Coxeter groups $\mathbf{5}$

2.1 Definition and examples . . . . . . . . . . . . 5

2.2 Basic combinatorial properties . . . . . . . . . . . . 7

2.2.1 The exchange and deletion properties . . . . . . . 7

2.2.2 The word property . . . . . . . . . . . . 8

2.3 The Bruhat order . . . . . . . . . . . . . . . . . 9

2.3.1 Parabolic subgroups and quotients . . . . . . . . 11

2.4 Twisted involutions and identities . . . . . . . . . . . . 12

2.4.1 A monoid action . . . . . . . . . . . . . . . 14

3 Poset topology 19

3.1 The order complex . . . . . . . . . . . . . . . 19

3.2 Piecewise linear topology . . . . . . . . . . . . . . . . . . 20

3.3 Shellability ......................... 21

3.3.1 Lexicographic shellability . . . . . . . . . . . 23

4 Combinatorics and topology related to involutions $\quad 27$

4.1 Topology of Bruhat orders . . . . . . . . . . . . . . 27

4.1.1 The Bruhat order on parabolic quotients . . . . . . . . 27

4.1.2 The Bruhat order on twisted involutions . . . . . . . . 28

4.1.3 The Bruhat order and zircons . . . . . . . . . . . . . . 29

4.1.4 The Bruhat order on twisted identities . . . . . . . . . 30

4.2 Word properties for twisted involutions . . . . . . . . . . 32

5 Summary of papers 35

5.1 Summary of Paper A . . . . . . . . . . . . . . . . . . 35

5.2 Summary of Paper B . . . . . . . . . . . . . . . . . . . . . . . . . . . . . . 36

5.3 Summary of Paper C . . . . . . . . . . . . . . . . . 39 
II Papers

Paper A 49

Paper B 65

Paper C 


\section{Part I}

\section{Introduction}





\section{Chapter 1}

\section{Posets}

In this chapter, we agree on poset terminology and notation. For the most part, we follow [42]. However, our definition of a graded poset is somewhat different. Throughout the dissertation, let $[n]=\{1,2, \ldots, n\}$ if $n$ is a nonnegative integer (so $[0]=\emptyset)$.

A poset (short for partially ordered set) is a set $P$ together with a binary relation $\leq$ on $P$ which is reflexive, antisymmetric, and transitive.

In a poset $P$, we say that $y$ covers $x$ if $x<y$ and there is no $z \in P$ such that $x<z<y$; we use the notation $x \triangleleft y$. Furthermore, a chain is a totally ordered subset, and a chain $C$ is saturated if there is no $z \in P-C$ such that $C \cup\{z\}$ is a chain and $x<z<y$ for some $x, y \in C$, and maximal if it is not contained in a larger chain. A $k$-chain is a chain of cardinality $k+1$. A $k$-chain $\left\{x_{0}<x_{1}<\cdots<x_{k}\right\}$ is saturated precisely when $x_{i-1} \triangleleft x_{i}$ for all $i \in[k]$. We call $P$ bounded if it has a minimum (denoted by $\hat{0}$ ) and a maximum (denoted by $\hat{1}$ ), which means that $\hat{0} \leq x$ and $\hat{1} \geq x$ for all $x \in P$. If $P$ is bounded, let its proper part be $\bar{P}=P-\{\hat{0}, \hat{1}\}$. An element $x \in P$ is minimal if there is no $y \in P$ with $y<x$, and maximal if there is no $y \in P$ with $y>x$. Observe that a minimal (maximal) element need not be a minimum (maximum).

An induced subposet of a poset $P$ is a subset of $P$ whose order relation is the restriction of that of $P$. In particular, if $x, y \in P$, then we view the closed interval $[x, y]=\{z \in P \mid x \leq z \leq y\}$ as an induced subposet of $P$, and similarly for open and half-open intervals. A principal order ideal in $P$ is an induced subposet of the form $\{x \in P \mid x \leq y\}$ for some $y \in P$.

A function $f: P \rightarrow Q$ of posets is called order-preserving if $x \leq y \Rightarrow$ $f(x) \leq f(y)$, and order-reversing if $x \leq y \Rightarrow f(x) \geq f(y)$. Two posets $P$ and $Q$ are isomorphic if there exists an order-preserving bijection $f: P \rightarrow Q$ whose inverse is also order-preserving; $f$ is then called an automorphism if $P=Q$. A bijection $f: P \rightarrow P$ is an anti-automorphism if $f$ and $f^{-1}$ are 
both order-reversing.

Given two posets $P$ and $Q$, let the direct product $P \times Q$ be the poset whose underlying set is the cartesian product $\{(p, q) \mid p \in P, q \in Q\}$, and where $(p, q) \leq\left(p^{\prime}, q^{\prime}\right)$ in $P \times Q$ if $p \leq p^{\prime}$ in $P$ and $q \leq q^{\prime}$ in $Q$. The dual of a poset has the same underlying set but the order relation is reversed.

There exist a few different, although very similar, definitions of pure, graded, and ranked posets in the literature. The terminology used throughout Part I and in Paper $\mathrm{C}$ is as follows. ${ }^{1}$ Call a poset $P$ pure if it is finite and every maximal chain has the same number of elements, and graded if every principal order ideal is pure. In this case, there exists a unique rank function $\rho: P \rightarrow\{0,1, \ldots\}$ such that $\rho(x)=0$ if $x$ is a minimal element of $P$, and $\rho(y)=\rho(x)+1$ if $x \triangleleft y$ in $P$.

\footnotetext{
${ }^{1}$ In Paper A, a graded poset is what we here call pure. However, when we there claim that some poset is not graded, we prove that there exists an interval with two maximal chains of different lengths. Hence, the poset is not graded also with the more permissive definition given here.
} 


\section{Chapter 2}

\section{Coxeter groups}

In the first three sections of this chapter, we present the basics of combinatorial Coxeter group theory that will be needed in the future. This and much more information can be found in [3] or [25]. In the last section, we introduce twisted involutions and list some of their fundamental properties.

\subsection{Definition and examples}

Let $S$ be a set. A Coxeter matrix is a function $m: S \times S \rightarrow\{1,2, \ldots, \infty\}$ such that $m\left(s, s^{\prime}\right)=m\left(s^{\prime}, s\right)$ for all $s, s^{\prime} \in S$, and $m\left(s, s^{\prime}\right)=1 \Leftrightarrow s=s^{\prime}$. Equivalently, $m$ can be represented by a Coxeter graph whose vertex set is $S$ and where $\left\{s, s^{\prime}\right\}$ is an edge if $m\left(s, s^{\prime}\right) \geq 3$, labelled by this number if it is greater than 3. Every Coxeter matrix $m$ determines a group $W$ with a presentation whose set of generators is $S$ and whose relations are $\left(s s^{\prime}\right)^{m\left(s, s^{\prime}\right)}=e$ whenever $m\left(s, s^{\prime}\right)<\infty$, where $e$ denotes the identity element of $W$. Since $m(s, s)=1, s^{2}=e$, so $m\left(s, s^{\prime}\right)=2$ precisely when $s$ and $s^{\prime}$ commute. The pair $(W, S)$ is called a Coxeter system; $W$ is the Coxeter group and $S$ is the set of Coxeter generators. The system is irreducible if its Coxeter graph is connected.

Let $S^{*}$ be the free monoid generated by $S$, i.e., the set of all words of finite length with letters from the alphabet $S$, with concatenation as product. Let $\equiv$ denote the equivalence relation generated by allowing insertion or deletion of any word of the form $s s^{\prime} s s^{\prime} \cdots s s^{\prime}$ of finite length $2 m\left(s, s^{\prime}\right)$. Then $S^{*} / \equiv$ forms a group isomorphic to $W$. It is a fact that if the generators $s$ and $s^{\prime}$ are distinct, then so are their equivalence classes, whence we may view $S$ as a subset of $W$. Furthermore, the order of the product $s s^{\prime}$ in $W$ is $m\left(s, s^{\prime}\right)$ (it is only obvious that the order divides $m\left(s, s^{\prime}\right)$ if $\left.m\left(s, s^{\prime}\right)<\infty\right)$. Therefore, there is a bijection between Coxeter graphs and Coxeter systems. 
Henceforth, we shall assume that $(W, S)$ is finitely generated, i.e., $S$ is finite, although much of what we do holds for arbitrary $S$.

We next describe two examples of Coxeter groups.

Example 2.1.1. The symmetric group. Consider the symmetric group $S_{n}$, i.e., the set of permutations of $[n]$. For each $i \in[n-1]$, let $s_{i}$ denote the adjacent transposition $(i, i+1)$, and let $S$ be the set of all $s_{i}$. It is clear that $S$ generates $S_{n}$. Furthermore, $s_{i} s_{j}=s_{j} s_{i}$ if $|i-j| \geq 2$ and $s_{i} s_{j} s_{i}=s_{j} s_{i} s_{j}$ if $|i-j|=1$. These facts make it conceivable that $\left(S_{n}, S\right)$ is the Coxeter system whose Coxeter graph is the path

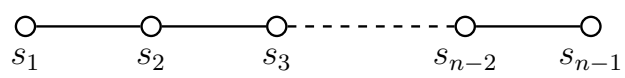

This also turns out to be the case. Thus, the symmetric group is an example of a Coxeter group. The Coxeter generators will always be the adjacent transpositions.

Example 2.1.2. Finite reflection groups. A finite reflection group is a finite group generated by reflections in some real euclidean space. What are the relations among the reflections? Some of them are obvious: those of the form $\left(s s^{\prime}\right)^{m\left(s, s^{\prime}\right)}=e$, where $m\left(s, s^{\prime}\right)$ is the order of $s s^{\prime}$. For $s \neq s^{\prime}, \pi / m\left(s, s^{\prime}\right)$ is the angle between the hyperplanes fixed pointwise by $s$ and $s^{\prime}$, respectively. It can be shown that these obvious relations completely determine the group: every finite reflection group is generated by a set $S$ of so-called simple reflections, subject only to the relations $\left(s s^{\prime}\right)^{m\left(s, s^{\prime}\right)}=e\left(s, s^{\prime} \in S\right)$. Thus every finite reflection group is a Coxeter group. The converse is, in fact, also true: every finite Coxeter group can be realised as a reflection group. The finite reflection groups for which $m\left(s, s^{\prime}\right) \in\{2,3,4,6\}$ for all $s \neq s^{\prime}$ in $S$ are called Weyl groups.

Reducible Coxeter groups decompose uniquely as products of irreducible ones; see Subsection 2.3.1. Thus, there is no essential loss of generality in restricting attention to the irreducible case. The finite, irreducible Coxeter systems and their Coxeter graphs are listed in Table 2.1 at the end of this chapter. Observe that $S_{n}$ is of type $A_{n-1}$ and that $I_{2}(3)=A_{2}$ and $I_{2}(4)=B_{2}$. The finite, irreducible Coxeter systems all determine distinct groups. This is not self-evident. In fact, if either condition is removed, it is no longer true. For instance, a group of type $I_{2}(6)$ is isomorphic to the direct product of two groups of types $A_{1}$ and $I_{2}(3)$. For an example of two non-isomorphic irreducible Coxeter systems which induce isomorphic groups, see [34]. 


\subsection{Basic combinatorial properties}

In this section, we present three fundamental combinatorial properties of Coxeter groups: the exchange, deletion, and word properties.

\subsubsection{The exchange and deletion properties}

Let $(W, S)$ be a Coxeter system. Suppose $w \in W$ is written as a product $w=s_{1} \cdots s_{k}$ of generators, and $w$ cannot be written as a product of fewer generators. Then $k$ is the length of $w$, and the word $s_{1} \cdots s_{k}$ is a reduced word, or a reduced expression, for $w$; let $\ell(w)$ denote the length of $w$. (Observe that we do not distinguish notationally between the group element $s_{1} \cdots s_{k}$ and the word $s_{1} \cdots s_{k}$.) For example, the length of a permutation $\sigma \in S_{n}$ is the number of inversions of $\sigma$, where an inversion is a pair $(i, j) \in[n]^{2}$ such that $i<j$ and $\sigma(i)>\sigma(j)$.

Given $w \in W$, let

$$
D_{R}(w)=\{s \in S \mid \ell(w s)<\ell(w)\}
$$

and

$$
D_{L}(w)=\{s \in S \mid \ell(s w)<\ell(w)\}
$$

be the sets of right descents and left descents, respectively. For all $w \in W$ and all $s \in S, \ell(w s)=\ell(w) \pm 1$ and $\ell(w)=\ell\left(w^{-1}\right)$, so $D_{R}(w)=D_{L}\left(w^{-1}\right)$ and $\ell(s w)=\ell(w) \pm 1$. Moreover, $\ell(w s)=\ell(w)-1$ if and only if some reduced word for $w$ ends with $s$, which is an immediate consequence of the following result.

Proposition 2.2.1 (Exchange property). Let $w \in W$. If $s_{1} \cdots s_{k}$ is a reduced word for $w$ and $s \in D_{R}(w)$, then $w s=s_{1} \cdots s_{i-1} s_{i+1} \cdots s_{k}$ for some $i \in[k]$.

From the exchange property, it is easy to derive the next result.

Proposition 2.2.2 (Deletion property). Suppose $w \in W$. If $s_{1} \cdots s_{k}$ is an unreduced word for $w$, then $w=s_{1} \cdots s_{i-1} s_{i+1} \cdots s_{j-1} s_{j+1} \cdots s_{k}$ for some $1 \leq i<j \leq k$.

Thus any expression for $w$ contains a reduced expression for $w$ as a subword, reachable by deleting an even number of letters. In particular, $S$ is a minimal generating set for $W$, i.e., no Coxeter generator can be expressed as a product of the others.

In fact, the exchange and deletion properties both characterise Coxeter groups among groups generated by involutions, a fact due to Matsumoto [33]. 
More precisely, assume $W$ is any group generated by a subset $S \subseteq W$ of elements of order 2 , and define length and reduced word as before. In the formulation of the exchange property, replace $s \in D_{R}(w)$ with $\ell(w s) \leq \ell(w)$. Then, if $(W, S)$ satisfies either the exchange or the deletion property, $(W, S)$ is Coxeter system.

There is a stronger version of the exchange property. According to [3], it was introduced in two unpublished papers by Tits and Verma, respectively. A proof can also be found in [3]. Given a Coxeter system $(W, S)$, let $T=$ $\left\{w s w^{-1} \mid s \in S, w \in W\right\}$ be the set of reflections. Note that $S \subseteq T$ and $t^{2}=e$ for all $t \in T$.

Proposition 2.2.3 (Strong exchange property). If $s_{1} \cdots s_{k}$ is any expression for $w$ and $\ell(w t)<\ell(w)$ for some $t \in T$, then $w t=s_{1} \cdots s_{i-1} s_{i+1} \cdots s_{k}$ for some $i \in[k]$.

\subsubsection{The word property}

The word problem is the problem of deciding whether two words represent the same group element. For Coxeter groups, there exists a solution based on the word property, discovered by Matsumoto [33] and Tits [43] in the 1960s. If $s s^{\prime}$ has finite order $m\left(s, s^{\prime}\right)$, a braid move is the replacement of $s s^{\prime} s \cdots$ by $s^{\prime} s s^{\prime} \cdots$, both consisting of $m\left(s, s^{\prime}\right)$ letters.

Theorem 2.2.4 (Word property). Let $(W, S)$ be a Coxeter system and let $w \in W$. Then any two reduced words for $w$ can be connected by a sequence of braid moves.

Example 2.2.5. Given a Coxeter system of type $B_{3}$ (see Table 2.1), consider the reduced words $s_{2} s_{1} s_{2} s_{1} s_{3} s_{2} s_{1}$ and $s_{1} s_{2} s_{1} s_{3} s_{2} s_{1} s_{3}$. They can be connected by the following sequence of braid moves:

$$
s_{2} s_{1} s_{2} s_{1} s_{3} s_{2} s_{1}=s_{1} s_{2} s_{1} s_{2} s_{3} s_{2} s_{1}=s_{1} s_{2} s_{1} s_{3} s_{2} s_{3} s_{1}=s_{1} s_{2} s_{1} s_{3} s_{2} s_{1} s_{3} .
$$

A direct consequence of the word property is that every reduced word for $w$ consists of the same letters from the alphabet $S$.

Given Theorem 2.2.4, it is not hard to prove that any expression (not necessarily reduced) for $w$ can be transformed into any reduced expression for $w$ by a sequence of braid moves and deletions of factors $s s$ for $s \in S$.

Corollary 2.2.6. Let $(W, S)$ be a Coxeter system and let $w \in W$. Then any word for $w$ can be transformed into any reduced word for $w$ via a sequence of braid moves and deletions of factors ss for $s \in S$. 
Example 2.2.7. Consider the expressions $s_{2} s_{1} s_{2} s_{3} s_{1} s_{3}$ and $s_{1} s_{2}$, where the latter is reduced, in a Coxeter system of type $A_{3}$. The former word can be transformed into the latter by the following sequence:

$$
s_{2} s_{1} s_{2} s_{3} s_{1} s_{3}=s_{1} s_{2} s_{1} s_{3} s_{1} s_{3}=s_{1} s_{2} s_{1} s_{1} s_{3} s_{3}=s_{1} s_{2} s_{3} s_{3}=s_{1} s_{2} .
$$

As a consequence, the word problem can be solved as follows. Given a word $\alpha \in S^{*}$, let $\pi(\alpha)$ be the set of all reduced words which can be obtained from $\alpha$ by some sequence of braid moves and deletions of factors ss. This is a finite set, computable in a finite number of steps. Clearly, if $\pi(\alpha) \cap \pi(\beta) \neq \emptyset$, then $\alpha$ and $\beta$ represent the same group element. Thanks to Corollary 2.2.6, the converse also holds.

\subsection{The Bruhat order}

The goal of this section is to define the Bruhat order and state some of its basic combinatorial properties.

Let $(W, S)$ be a Coxeter system. The Bruhat order is a partial order $\leq$ on $W$ defined as follows.

Definition 2.3.1. Let $u, w \in W$. Write $u \rightarrow w$ if $u^{-1} w \in T$ and $\ell(u)<\ell(w)$, where $T=\left\{w s w^{-1} \mid s \in S, w \in W\right\}$ is the set of reflections. Then $u \leq w$ if $u=w$ or $u \rightarrow \cdots \rightarrow w$.

It is clear that $\leq$ is a partial order, $u<w \Rightarrow \ell(u)<\ell(w), u<u t \Leftrightarrow$ $\ell(u)<\ell(u t)$ for $t \in T$, and $e \leq w$ for all $w \in W$ (if $s_{1} \cdots s_{k}$ is a reduced word for $w$, then $\left.e \rightarrow s_{1} \rightarrow s_{1} s_{2} \rightarrow \cdots \rightarrow w\right)$. See Figure 2.3.1 for the Hasse diagram of the Bruhat order on $S_{3}$.

The following important result of Chevalley [8] characterises the Bruhat order.

Theorem 2.3.2 (Subword property). Let $u, w \in W$ and assume $s_{1} \cdots s_{k}$ is a reduced word for $w$. Then $u \leq w$ in the Bruhat order if and only if $s_{i_{1}} \cdots s_{i_{m}}$ is a reduced word for $u$, for some $1 \leq i_{1}<\cdots<i_{m} \leq k$.

Corollary 2.3.3. For $u, w \in W$, the following statements are equivalent:

- $u \leq w$;

- every reduced word for w has a subword that is a reduced word for u;

- some reduced word for $w$ has a subword that is a reduced word for $u$.

It follows from the subword property that every Bruhat interval $[u, w]$ is finite and that the function $w \mapsto w^{-1}$ is an automorphism of the Bruhat 


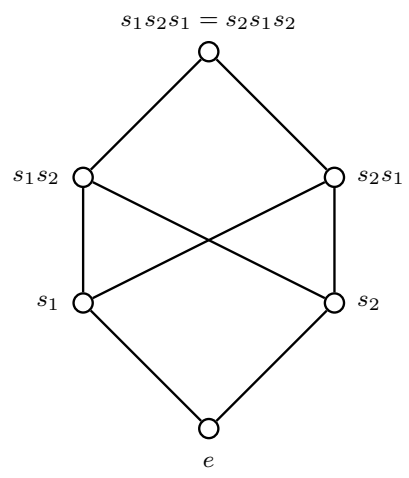

(a)

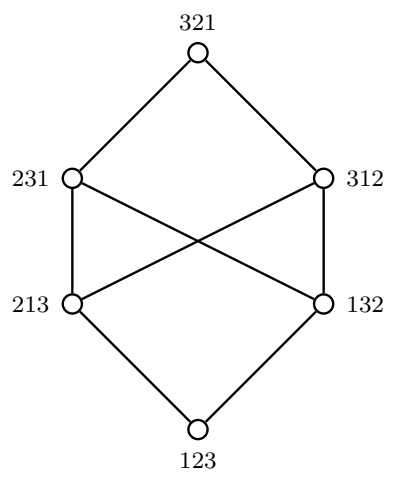

(b)

Figure 2.3.1: The Bruhat order on $S_{3}$. In (a), the permutations are written as reduced words, with $s_{i}=(i, i+1)$. In (b), they are written in one-line notation.

order. Moreover, it turns out that the Bruhat order is a graded poset whose rank function is the length function.

There is a simple characterisation of the Bruhat order on $S_{n}$. For $\sigma \in S_{n}$ and $(k, l) \in[n]^{2}$, let $\sigma[k, l]=|\{i \leq k \mid \sigma(i) \geq l\}|$.

Theorem 2.3.4. Let $\sigma, \tau \in S_{n}$. Then $\sigma \leq \tau$ in the Bruhat order if and only if $\sigma[k, l] \leq \tau[k, l]$ for all $(k, l) \in[n]^{2}$.

Example 2.3.5. In $S_{5}$, we have $34251>21435$ (one-line notation). On the other hand, $\sigma=34251$ and $\tau=21534$ are incomparable in the Bruhat order since $\sigma[1,3]>\tau[1,3]$ and $\sigma[3,5]<\tau[3,5]$.

The next result, due to Deodhar [10], is often useful.

Proposition 2.3.6 (Lifting property). If $u \leq w$ in the Bruhat order on $W$ and $s \in D_{R}(w)$, then

(i) $u s \leq w$,

(ii) $s \in D_{R}(u) \Rightarrow u s \leq w s$, and

(iii) $s \notin D_{R}(u) \Rightarrow u \leq w s$.

The lifting property can be used to prove that the Bruhat order is a directed poset, i.e., for all $u, w \in W$ there exists an element $z \in W$ such that $u, w \leq z$. If $W$ is finite, this means that $W$ has a maximum, denoted $w_{0}$. Since $\ell\left(w_{0}\right)=\ell\left(w_{0}^{-1}\right), w_{0}^{2}=e$. Clearly, $D_{R}\left(w_{0}\right)=S$. Conversely, it can be 
shown that if $D_{R}(w)=S$ for some $w \in W$, then $w=w_{0}$, whence $W=\left[e, w_{0}\right]$ is finite.

Let us briefly describe a classic, geometric motivation for studying the Bruhat order. Denote by $\mathrm{Fl}_{n}$ the set of all sequences $\{0\} \subset V_{1} \subset V_{2} \subset \cdots \subset$ $V_{n-1} \subset \mathbf{C}^{n}$ of linear subspaces, where $\operatorname{dim}_{\mathbf{C}} V_{i}=i$ for all $i \in[n-1]$; the sequences are called flags. There is a bijection between $\mathrm{Fl}_{n}$ and $G / B$, where $G=\mathrm{GL}_{n}(\mathbf{C})$ and $B$ is the subgroup of upper-triangular matrices. It is a fact that $G / B$ is the disjoint union

$$
G / B=\biguplus_{w \in S_{n}} B w B / B
$$

where we identify a permutation $w \in S_{n}$ with the corresponding permutation matrix. We call $C_{w}=B w B / B$ a Schubert cell (or a Bruhat cell); the word "cell" is due to the fact that one may define a topology on $\mathrm{Fl}_{n}$ under which each $C_{w}$ is a cell (i.e., homeomorphic to an open ball). The topological closure of a Schubert cell $C_{w}$ is called a $S$ chubert variety; we use the notation $\overline{C_{w}}$. It turns out that

$$
\overline{C_{w}}=\biguplus_{v \leq w} C_{v},
$$

where $\leq$ is the Bruhat order on $S_{n}$. Hence, $\overline{C_{u}} \subseteq \overline{C_{w}}$ precisely when $u \leq w$.

\subsubsection{Parabolic subgroups and quotients}

Let $(W, S)$ be a Coxeter system. A parabolic subgroup $W_{J}$ of $W$ is a subgroup generated by some subset $J \subseteq S$. In particular, $W_{\emptyset}=\{e\}$ and $W_{S}=W$. It is a fact that $\left(W_{J}, J\right)$ is a Coxeter system whose length function agrees with $\ell$ on $W_{J}$, and $I=J \Leftrightarrow W_{I}=W_{J}$. Furthermore, the Coxeter graph of $\left(W_{J}, J\right)$ is the induced subgraph on $J$ of the Coxeter graph of $(W, S)$, and the Bruhat order on the Coxeter group $W_{J}$ coincides with the Bruhat order on $W$ restricted to $W_{J}$. Moreover, if $J_{1}, \ldots, J_{k}$ are the vertex sets of the connected components of the Coxeter graph of $(W, S)$, then $W \cong$ $W_{J_{1}} \times \cdots \times W_{J_{k}}$ as groups and $\operatorname{Br}(W) \cong \operatorname{Br}\left(W_{J_{1}}\right) \times \cdots \times \operatorname{Br}\left(W_{J_{k}}\right)$ as posets, where $\operatorname{Br}(X)$ denotes the induced Bruhat order on $X \subseteq W$.

For $J \subseteq S$, let $W^{J}=\{w \in W \mid w s>w$ for all $s \in J\}$. In particular, $W^{\emptyset}=W$ and $W^{S}=\{e\}$. Note that $e \in W^{J}$ for all $J \subseteq S$.

Proposition 2.3.7. Let $J \subseteq S$. Every $w \in W$ has a unique factorisation $w=w^{J} w_{J}$ such that $w^{J} \in W^{J}$ and $w_{J} \in W_{J}$. Moreover, $\ell(w)=\ell\left(w^{J}\right)+\ell\left(w_{J}\right)$ and $w^{J}$ is the unique element of minimal length in the coset $w W_{J}$. 
So, $W^{J}=\left\{w \in W \mid \ell(w)\right.$ is minimal in $\left.w W_{J}\right\}$. The set $W^{J}$ of minimal coset representatives is called a parabolic quotient.

The function $W \rightarrow W^{J}$ defined by $w \mapsto w^{J}$ is order-preserving. Since $\operatorname{Br}(W)$ is directed, so is $\operatorname{Br}\left(W^{J}\right)$. In particular, $W^{J}$ has a maximum if it is finite. However, if $(W, S)$ is infinite and irreducible, then $W^{J}$ is infinite for all proper subsets $J \subset S$; see [11]. The Bruhat order on $W^{J}$ is a graded poset whose rank function is the length function.

\subsection{Twisted involutions and identities}

The results of this section are taken from Hultman's papers [21, 22, 24]. Many of the results can be found for finite Coxeter groups already in [38, 39, 41] by Richardson and Springer.

Let $(W, S)$ be a Coxeter system with an involutive automorphism $\theta$ (i.e., a group automorphism $\theta$ of $W$ such that $\theta(S)=S$ and $\theta^{2}=\mathrm{id}$ ). In other words, $\theta$ is induced by an involutive automorphism of the Coxeter graph of $(W, S)$. Define the sets of twisted involutions and twisted identities as

$$
\mathfrak{I}(\theta)=\left\{w \in W \mid \theta(w)=w^{-1}\right\}
$$

and

$$
\iota(\theta)=\left\{\theta\left(w^{-1}\right) w \mid w \in W\right\},
$$

respectively. Note that $\{e\} \subseteq \iota(\theta) \subseteq \mathfrak{I}(\theta)$. Furthermore, $\mathfrak{I}(\mathrm{id})$ is the set of ordinary involutions in $W$ while $\iota(\mathrm{id})=\{e\}$. Observe that $e$ is the minimum of $\operatorname{Br}(\mathfrak{I}(\theta))$ and $w_{0}$ is the maximum if $W$ is finite. Figures 2.4.1 and 2.4.2 depict the Hasse diagrams of $\operatorname{Br}(W)$ and $\operatorname{Br}(\mathfrak{I}(\theta))$ when $W$ is of type $B_{2}=$ $I_{2}(4)$. In this case, $\iota(\theta)=\mathfrak{I}(\theta)$ when $\theta \neq \mathrm{id}$. Note that some Coxeter systems have no non-trivial $\theta$, while others have more than one.

Systematic study of $\mathfrak{I}(\theta)$ was initiated by Richardson and Springer in the 1980s. Their main motivation was its connections with Borel orbit decompositions of symmetric varieties. As in the motivating example in Section 2.3, let $G=\mathrm{GL}_{n}(\mathbf{C})$ and let $B$ be the subgroup of upper-triangular matrices. Suppose $\Theta$ is an automorphism of $G$ of order 2 which preserves $B$ and the subgroup of diagonal matrices, and $K$ is its fixed point subgroup. Then $B \backslash G / K$ can be ordered by containment of topological closures, as before. In order to study this poset, Richardson and Springer defined an order-preserving map $\varphi: B \backslash G / K \rightarrow \operatorname{Br}(\mathfrak{I}(\theta)) \subseteq \operatorname{Br}\left(S_{n}\right)$, where $\theta$ is induced by $\Theta$.

This is done also for other groups $G$, giving rise to maps $\varphi: B \backslash G / K \rightarrow$ $\operatorname{Br}(\mathfrak{I}(\theta)) \subseteq \operatorname{Br}(W)$, where $W$ is a Weyl group. In general, $\varphi$ is neither injective nor surjective. However, $\iota(\theta)$ is always contained in the image. More- 


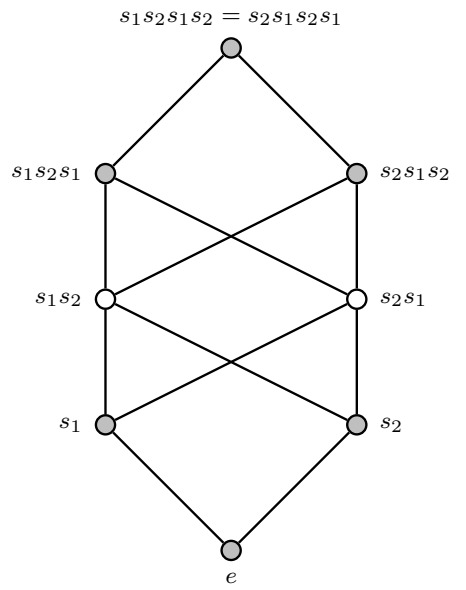

$\theta=\mathrm{id}$

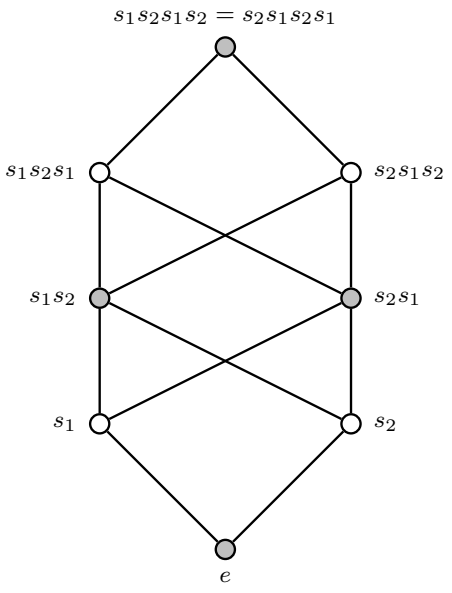

$\theta \neq \mathrm{id}$

Figure 2.4.1: The Bruhat order in type $B_{2}$ with the elements of $\mathfrak{I}(\theta)$ shaded.

over, there exist examples where $\varphi$ induces a poset isomorphism $B \backslash G / K \rightarrow$ $\operatorname{Br}(\mathfrak{I}(\theta))$ or $B \backslash G / K \rightarrow \operatorname{Br}(\iota(\theta))$.

(Bruhat orders on) twisted involutions and identities generalise (Bruhat orders on) Coxeter groups, as the following example shows.

Example 2.4.1. Let $W$ be any Coxeter group, and consider the involution $\theta: W \times W \rightarrow W \times W$ given by $(u, w) \mapsto(w, u)$. Then

$$
\iota(\theta)=\mathfrak{I}(\theta)=\left\{\left(w, w^{-1}\right) \mid w \in W\right\},
$$

so there exists a bijection $W \rightarrow \iota(\theta)=\mathfrak{I}(\theta)$ given by $w \mapsto\left(w, w^{-1}\right)$. It follows from the subword property (Theorem 2.3.2) that it is a poset isomorphism, so that $\operatorname{Br}(\iota(\theta)) \cong \operatorname{Br}(W)$ and $\operatorname{Br}(\mathfrak{I}(\theta)) \cong \operatorname{Br}(W)$.

In Example 2.4.1, $\iota(\theta)=\mathfrak{I}(\theta)$. This is always the case if there exists no $s \in S$ such that $\theta(s)$ and $s$ are conjugate.

It turns out that $\operatorname{Br}(\mathfrak{I}(\theta))$ is always graded. In order to describe its rank function, let the twisted absolute length (with respect to $\theta) \ell^{\theta}(w)$ of $w \in W$ be the smallest number of generators that must be deleted from a reduced expression for $w$ in order to obtain a twisted identity. Although not obvious, $\ell^{\theta}(w)$ is independent of the choice of reduced word for $w$, hence is well defined. The absolute length of $w$ is $\ell^{\prime}(w)=\min \left\{k \mid w=t_{1} \cdots t_{k}\right.$ with $\left.t_{1}, \ldots, t_{k} \in T\right\}$, where $T$ is the set of reflections. Because $\ell^{\prime}(w)$ is also equal to the smallest 


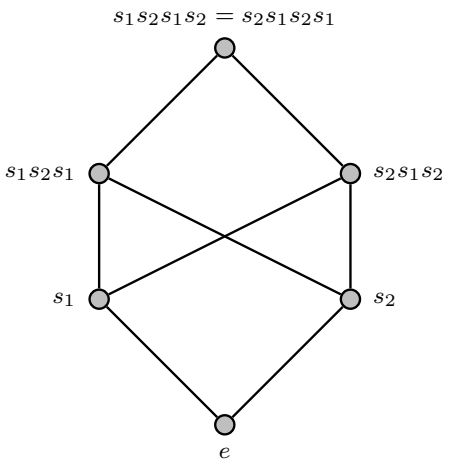

$\theta=\mathrm{id}$

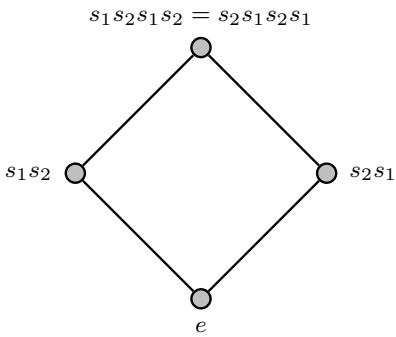

$\theta \neq$ id

Figure 2.4.2: The induced subposet $\operatorname{Br}(\Im(\theta))$ in type $B_{2}$.

number of generators that must be deleted from a reduced expression for $w$ in order to obtain $e$, as shown by Dyer [15], $\ell^{\text {id }}=\ell^{\prime}$.

Theorem 2.4.2. The rank of $w \in \operatorname{Br}(\mathfrak{I}(\theta))$ is $\left(\ell(w)+\ell^{\theta}(w)\right) / 2$.

In particular, observe that the rank function of $\operatorname{Br}(\mathfrak{I}(\theta))$ does not agree with the restriction of the rank function of $\operatorname{Br}(W)$. This stands in contrast to the situation for $\operatorname{Br}\left(W^{J}\right), J \subseteq S$, whose rank function does agree with the restriction of the rank function of $\operatorname{Br}(W)$.

\subsubsection{A monoid action}

Like elements of $W$, twisted involutions can be represented by "words" and "reduced expressions", this time in the alphabet $\underline{S}=\{\underline{s} \mid s \in S\}$. More precisely, we have the following definition.

Definition 2.4.3. The free monoid $\underline{S}^{*}$ acts from the right on the set $W$ by

$$
w \underline{s}= \begin{cases}w s & \text { if } \theta(s) w s=w \\ \theta(s) w s & \text { otherwise }\end{cases}
$$

and $w \underline{s}_{1} \cdots \underline{s}_{k}=\left(\cdots\left(\left(w \underline{s}_{1}\right) \underline{s}_{2}\right) \cdots\right) \underline{s}_{k}$. By abuse of notation, we write $\underline{s}_{1} \cdots \underline{s}_{k}$ for $e \underline{s}_{1} \cdots \underline{s}_{k}$, where $e \in W$ is the identity element.

Observe that $w \underline{s} \underline{s}=w$ for all $w \in W$ and all $s \in S$. The orbit of $e$ under this action is precisely $\mathfrak{I}(\theta)$ : 
Proposition 2.4.4. We have

$$
\mathfrak{I}(\theta)=\left\{\underline{s}_{1} \cdots \underline{s}_{k} \mid \underline{s}_{1}, \ldots, \underline{s}_{k} \in \underline{S}, k \in \mathbf{N}\right\} .
$$

That is, every word in $\underline{S}^{*}$ represents a twisted involution, and conversely, every twisted involution can be represented in this way.

Example 2.4.5. Let us illustrate Definition 2.4.3 and Proposition 2.4.4 in the case of type $B_{2}$ with $\theta \neq$ id; see Figure 2.4.2.

- Since $\theta\left(s_{1}\right) e s_{1}=s_{2} e s_{1} \neq e$, we have $\underline{s}_{1}=e \underline{s}_{1}=s_{2} e s_{1}=s_{2} s_{1}$; similarly, $\underline{s}_{2}=s_{1} s_{2}$.

- We have $\underline{s}_{1} \underline{s}_{2}=s_{2} s_{1} \underline{s}_{2}=s_{1} s_{2} s_{1} s_{2}$ because $\theta\left(s_{2}\right)\left(s_{2} s_{1}\right) s_{2}=s_{1} s_{2} s_{1} s_{2} \neq$ $s_{2} s_{1}$; similarly, $\underline{s}_{2} \underline{s}_{1}=s_{2} s_{1} s_{2} s_{1}$, so $\underline{s}_{1} \underline{s}_{2}=\underline{s}_{2} \underline{s}_{1}$.

- Since $\underline{s}_{1} \underline{s}_{2}=\underline{s}_{2} \underline{s}_{1}, \underline{s}_{1} \underline{s}_{2} \underline{s}_{1}=\underline{s}_{2} \underline{s}_{1} \underline{s}_{1}=\underline{s}_{2}$. Thus, $\underline{s}_{1} \underline{s}_{2} \underline{s}_{1} \underline{s}_{2}=\underline{s}_{2} \underline{s}_{2}=e$.

As for Coxeter groups, $\underline{s}_{1} \cdots \underline{s}_{k}$ can denote both an element of $\mathfrak{I}(\theta)$ and an element of $\underline{S}^{*}$. If $w=\underline{s}_{1} \cdots \underline{s}_{k}$ for some $\underline{s}_{i} \in \underline{S}$, the word $\underline{s}_{1} \cdots \underline{s}_{k}$ is called an $\underline{S}$-expression for $w$. It is reduced if no $\underline{S}$-expression for $w$ consists of fewer than $k$ elements of $\underline{S}$. In this case, $\rho(w)=k$ is the rank of $w$. It coincides with the rank function of Theorem 2.4.2, i.e., $\rho=\left(\ell+\ell^{\theta}\right) / 2$. Observe that a consequence is the following characterisation of $\iota(\theta)$ :

$$
\iota(\theta)=\left\{w \in \mathfrak{I}(\theta) \mid \ell^{\theta}(w)=0\right\}=\{w \in \mathfrak{I}(\theta) \mid \ell(w)=2 \rho(w)\} .
$$

The combinatorics of $\mathfrak{I}(\theta)$ is in many regards strikingly similar to that of $W$. For example, $\rho(w \underline{s})=\rho(w) \pm 1$ and $\rho(w \underline{s})<\rho(w) \Leftrightarrow \ell(w s)<\ell(w)$. Moreover, the exchange, deletion, lifting, and subword properties have the following analogues.

Proposition 2.4.6 (Exchange property for $\mathfrak{I}(\theta)$ ). Let $w \in \mathfrak{I}(\theta)$. If $\underline{s}_{1} \cdots \underline{s}_{k}$ is a reduced $\underline{S}$-expression for $w$ and $s \in D_{R}(w)$, then $w \underline{s}=\underline{s}_{1} \cdots \underline{s}_{i-1} \underline{s}_{i+1} \cdots \underline{s}_{k}$ for some $i \in[k]$.

Proposition 2.4.7 (Deletion property for $\mathfrak{I}(\theta)$ ). Let $w \in \mathfrak{I}(\theta)$. If $\underline{s}_{1} \cdots \underline{s}_{k}$ is an unreduced $\underline{S}$-expression for $w$, then $w=\underline{s}_{1} \cdots \underline{s}_{i-1} \underline{s}_{i+1} \cdots \underline{s}_{j-1} \underline{s}_{j+1} \cdots \underline{s}_{k}$ for some $1 \leq i<j \leq k$.

Proposition 2.4.8 (Lifting property for $\mathfrak{I}(\theta)$ ). If $u \leq w$ in $\operatorname{Br}(\mathfrak{I}(\theta)$ ) and $s \in D_{R}(w)$, then

(i) $u \underline{s} \leq w$,

(ii) $s \in D_{R}(u) \Rightarrow u \underline{s} \leq w \underline{s}$, and

(iii) $s \notin D_{R}(u) \Rightarrow u \leq w \underline{s}$. 
Proposition 2.4.8 can be used to prove that $\operatorname{Br}(\mathfrak{I}(\theta))$ is directed, just as Proposition 2.3.6 can be used to prove directedness of $\operatorname{Br}(W)$.

Theorem 2.4.9 (Subword property for $\mathfrak{I}(\theta)$ ). Let $u, w \in \mathfrak{I}(\theta)$ and assume $\underline{s}_{1} \cdots \underline{s}_{k}$ is a reduced $\underline{S}$-expression for $w$. Then $u \leq w$ in the Bruhat order if and only if $\underline{s}_{i_{1}} \cdots \underline{s}_{i_{m}}$ is a reduced $\underline{S}$-expression for $u$, for some $1 \leq i_{1}<$ $\cdots<i_{m} \leq k$.

Corollary 2.4.10. For $u, w \in \mathfrak{I}(\theta)$, the following statements are equivalent:

- $u \leq w$;

- every reduced $\underline{S}$-expression for $w$ has a subword that is a reduced $\underline{S}$ expression for $u$;

- some reduced $\underline{S}$-expression for $w$ has a subword that is a reduced $\underline{S}$ expression for $u$. 


\begin{tabular}{|c|c|}
\hline Type & Coxeter graph \\
\hline$A_{n}(n \geq 1)$ & 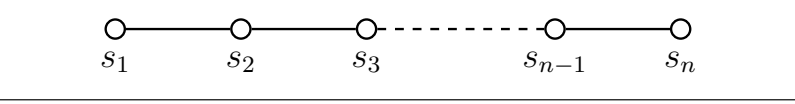 \\
\hline$B_{n}(n \geq 2)$ & 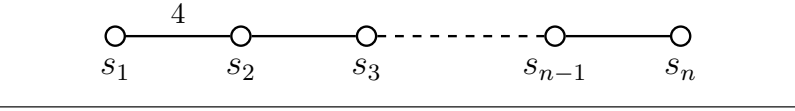 \\
\hline$D_{n}(n \geq 4)$ & $\begin{array}{ccc}- & - & - \\
s_{4} & s_{n-1} & s_{n}\end{array}$ \\
\hline$E_{6}$ & $\mathrm{O}$ \\
\hline$E_{7}$ & $\mathrm{O}-\mathrm{O}-\mathrm{O}-\mathrm{O}-\mathrm{O}-\mathrm{O}$ \\
\hline$E_{8}$ & 0 \\
\hline$F_{4}$ & $\mathrm{O}$ \\
\hline$H_{3}$ & $\mathrm{O}$ \\
\hline$H_{4}$ & $\mathrm{O}$ \\
\hline$I_{2}(m)(m \geq 3)$ & $\circ$ \\
\hline
\end{tabular}

Table 2.1: The finite, irreducible Coxeter systems. 



\section{Chapter 3}

\section{Poset topology}

Papers $\mathrm{A}$ and $\mathrm{C}$ deal with the combinatorial structure of (intervals in) posets, notably various induced subposets of $\operatorname{Br}(W)$ for Coxeter groups $W$. In Paper $\mathrm{C}$ in particular, we investigate the topology of certain posets, by which we mean the topology of their order complexes. The goal of this chapter is to define this complex and the notion of shellability and to give a very brief introduction to piecewise linear topology.

\subsection{The order complex}

In this section, we define the order complex of a poset. We first have to define simplicial complexes, which we shall assume to be finite. Everything can be found in [32], where also some topological notions are reviewed.

Definition 3.1.1. Let $V$ be a finite set whose elements are called vertices. An abstract simplicial complex $\Delta$ on $V$ is a family of subsets of $V$ such that

- $\{v\} \in \Delta$ for all $v \in V$, and

- if $F \subseteq F^{\prime} \in \Delta$, then $F \in \Delta$.

We call the elements of $\Delta$ its simplices or faces, and facets are maximal faces. The dimension of a face $F$ is $|F|-1 ; F$ is a $d$-face if its dimension is $d$. If $\Delta \neq \emptyset$, its dimension, denoted by $\operatorname{dim} \Delta$, is the maximum of the dimensions of the facets of $\Delta$. We call $\Delta$ pure if all facets have the same dimension.

Definition 3.1.2. For $d \geq-1$, a $d$-dimensional geometric simplex $\sigma$ is the convex hull of $d+1$ affinely independent points (called the vertices of $\sigma$ ) in some $\mathbf{R}^{n}$. The convex hull of any subset of the vertices is also a simplex, called a face of $\sigma$.

A geometric simplicial complex $\Delta$ is a finite collection of geometric simplices in some $\mathbf{R}^{n}$ such that 
- if $\tau \in \Delta$ and $\sigma$ is a face of $\tau$, then $\sigma \in \Delta$, and

- if $\sigma, \tau \in \Delta$, then $\sigma \cap \tau$ is a face of both $\sigma$ and $\tau$.

The geometric realisation $\|\Delta\|$ of $\Delta$ is the union of the simplices of $\Delta$. We view it as a topological space, with the subspace topology induced by the usual topology on $\mathbf{R}^{n}$.

Let $V(\Delta)$ denote the union of the vertex sets of all geometric simplices of $\Delta$. If $f: V(\Delta) \rightarrow V\left(\Delta^{\prime}\right)$ is a function such that conv $\left\{f\left(v_{0}\right), \ldots, f\left(v_{d}\right)\right\} \in \Delta^{\prime}$ whenever conv $\left\{v_{0}, \ldots, v_{d}\right\} \in \Delta$, then $f$ can be extended to a continuous function $\|f\|:\|\Delta\| \rightarrow\left\|\Delta^{\prime}\right\|$ defined by $\|f\|\left(\sum_{i=0}^{d} a_{i} v_{i}\right)=\sum_{i=0}^{d} a_{i} f\left(v_{i}\right)$, where $a_{0}, \ldots, a_{d} \geq 0$ and $\sum_{i=0}^{d} a_{i}=1$. We call $\|f\|$ the (linear) simplicial map induced by $f$. In case $f$ is a bijection such that conv $\left\{v_{0}, \ldots, v_{d}\right\} \in \Delta \Leftrightarrow$ $\operatorname{conv}\left\{f\left(v_{0}\right), \ldots, f\left(v_{d}\right)\right\} \in \Delta^{\prime}$, then $\|f\|$ is a homeomorphism.

Each geometric simplicial complex determines an abstract one: let the faces of the latter be the vertex sets of the faces of the former. Every abstract simplicial complex can be obtained in this way, and the geometric realisation of the corresponding geometric simplicial complexes is unique up to linear homeomorphism.

Every finite poset defines an abstract simplicial complex, as follows:

Definition 3.1.3. Given a finite poset $P$, let the order complex of $P$ be the abstract simplicial complex $\Delta(P)$ whose vertices are the elements of $P$ and whose faces are the chains in $P$.

Note that the facets of $\Delta(P)$ are the maximal chains in $P$. Hence, $\Delta(P)$ is pure if and only if $P$ is pure.

\subsection{Piecewise linear topology}

In this section, we define the basic notions from piecewise linear topology that are used in the remainder of Part I. General references are [20, 40].

Suppose $\Delta_{1}$ and $\Delta_{2}$ are abstract simplicial complexes. Then $\Delta_{1}$ is called a simplicial subdivision of $\Delta_{2}$ if there exist corresponding geometric simplicial complexes $\Delta_{1}^{\prime}$ and $\Delta_{2}^{\prime}$ such that $\left\|\Delta_{1}^{\prime}\right\|=\left\|\Delta_{2}^{\prime}\right\|$ and each simplex of $\Delta_{1}^{\prime}$ is contained in a simplex of $\Delta_{2}^{\prime}$. An example is provided by $\Delta_{1}=$ $\{\emptyset,\{a\},\{b\},\{c\},\{a, b\},\{b, c\}\}$ and $\Delta_{2}=\{\emptyset,\{x\},\{y\},\{x, y\}\}$.

Let $X$ be a topological space. A geometric simplicial complex $\Delta$ such that $\|\Delta\|$ is homeomorphic to $X$ is called a triangulation of $X$. For example, any geometric simplicial complex corresponding to $\Delta_{1} \cup\{\{a, c\}\}$, with $\Delta_{1}$ as above, is a triangulation of the 1-sphere. 
Definition 3.2.1. Suppose $\Delta$ and $\Delta^{\prime}$ are abstract simplicial complexes. A continuous map $f:\|\Delta\| \rightarrow\left\|\Delta^{\prime}\right\|$ is piecewise linear, or $P L$ for short, if there exist simplicial subdivisions $\tilde{\Delta}$ and $\tilde{\Delta}^{\prime}$ of $\Delta$ and $\Delta^{\prime}$, respectively, such that $f$ is a simplicial map of the corresponding triangulations of $\|\Delta\|$ and $\left\|\Delta^{\prime}\right\|$. We say that $\Delta$ and $\Delta^{\prime}$ are $P L$ homeomorphic if there exists a PL homeomorphism $f:\|\Delta\| \rightarrow\left\|\Delta^{\prime}\right\|$.

If $f:\|\Delta\| \rightarrow\left\|\Delta^{\prime}\right\|$ is a PL homeomorphism, then so is $f^{-1}$. Two abstract simplicial complexes $\Delta$ and $\Delta^{\prime}$ are PL homeomorphic if and only if there exist simplicial subdivisions $\tilde{\Delta}$ and $\tilde{\Delta}^{\prime}$ which are isomorphic.

Henceforth, we shall refer to abstract simplicial complexes simply as simplicial complexes.

For $d \geq 0$, call a simplicial complex a $P L d$-ball if it is PL homeomorphic to the simplicial complex $\Delta^{d}$ whose only facet is the $d$-simplex, and let a $P L(d-1)$-sphere be a simplicial complex which is PL homeomorphic to the simplicial complex obtained by removing the facet from $\Delta^{d}$.

Given a simplicial complex $\Delta$ and a face $\sigma \in \Delta$, let the link of $\sigma$ be the subcomplex $\operatorname{lk}_{\Delta}(\sigma)=\{\tau \in \Delta \mid \sigma \cap \tau=\emptyset$ and $\sigma \cup \tau \in \Delta\}$. The closure $\operatorname{cl}(\mathcal{F})$ of a finite family $\mathcal{F}$ of finite sets is the simplicial complex generated by $\mathcal{F}$, i.e., $\operatorname{cl}(\mathcal{F})=\{\sigma \mid \sigma \subseteq F$ for some $F \in \mathcal{F}\}$.

A $P L d$-manifold is a $d$-dimensional simplicial complex such that, for all $k \geq 0$, the link of every $k$-face is a PL $(d-1-k)$-ball or sphere. If $\Delta$ is a PL $d$-manifold, its boundary $\partial \Delta$ is the simplicial complex whose facets are the $(d-1)$-faces of $\Delta$ that are contained in only one facet of $\Delta$. PL $d$-balls are PL $d$-manifolds with PL $(d-1)$-spheres as boundaries, while PL $d$-spheres are PL $d$-manifolds without boundaries.

The importance of the PL condition lies in the fact that several desirable and seemingly plausible properties of balls and spheres would be false without it. One such example is the following: The union of two PL $d$-balls, whose intersection is a PL $(d-1)$-ball contained in the boundaries of both, is a $\mathrm{PL} d$-ball. Another example is provided by Newman's theorem: The closure of the complement of a PL $d$-ball embedded in a PL $d$-sphere is a PL $d$ ball. In case "PL $d$-ball" were replaced by " $d$-ball", i.e., a simplicial complex which is homeomorphic to $\Delta^{d}$, and similarly for "PL $d$-sphere", then the two statements above would be false. For details, see [29] and the references listed therein.

\subsection{Shellability}

The first goal of this section, which is based on [44], is to introduce the important concept of a shellable simplicial complex. We shall then record 


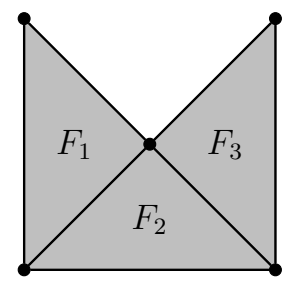

(a)

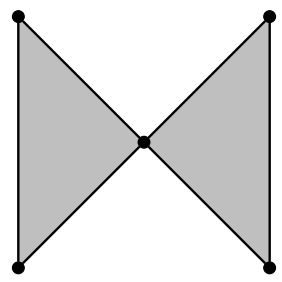

(b)

Figure 3.3.1: A shellable and a non-shellable simplicial complex. In (a), $F_{1}<F_{2}<F_{3}$ is a shelling order whereas $F_{1}<F_{3}<F_{2}$ is not.

some of the main consequences of shellability for the topology of the complex, and see how one can establish shellability of order complexes.

Let $\Delta$ be a simplicial complex. If $\tau \in \Delta$, let $[\tau]=\operatorname{cl}(\{\tau\})=\{\sigma \mid \sigma \subseteq \tau\}$.

Definition 3.3.1. Given a simplicial complex $\Delta$, let $\mathcal{F}=\left\{F_{1}, \ldots, F_{m}\right\}$ be its set of facets. A shelling order of $\Delta$ is a total order on $\mathcal{F}$, say $F_{1}<\cdots<F_{m}$, such that

$$
\left(\bigcup_{i=1}^{k-1}\left[F_{i}\right]\right) \cap\left[F_{k}\right]
$$

is a pure subcomplex of $\left[F_{k}\right]$ of codimension 1 (i.e., of dimension $\operatorname{dim} F_{k}-1$ ), for all $2 \leq k \leq m$. If $\Delta$ has a shelling order, $\Delta$ is called shellable.

The concepts of Definition 3.3.1 are illustrated in Figure 3.3.1.

A wedge of finitely many mutually disjoint topological spaces is obtained by choosing a base point for each space and then identifying all the base points. The homeomorphism type, and in particular the homotopy type, of a wedge of spheres is independent of the choice of base points.

Given a shelling order $F_{1}<\cdots<F_{m}$, call $F_{k}$ a homology facet if

$$
\left(\bigcup_{i=1}^{k-1}\left[F_{i}\right]\right) \cap\left[F_{k}\right]
$$

is the entire boundary of $\left[F_{k}\right]$.

A topological consequence of shellability is the following result of Björner and Wachs.

Theorem 3.3.2 ([5]). If $\Delta$ is a shellable simplicial complex, then $\Delta$ has the homotopy type of a wedge of spheres, where for each $d$, the number of $d$-spheres is equal to the number of homology d-facets. In particular, if $\Delta$ is pure, then $\Delta$ is homotopy equivalent to a wedge of spheres of dimension $\operatorname{dim} \Delta$. 
As a corollary, observe that the number of homology $d$-facets of a shellable simplicial complex is independent of the shelling order. The shelling order $F_{1}<F_{2}<F_{3}$ of the simplicial complex $\Delta$ given in Figure 3.3.1(a) has zero homology facets, so it has the homotopy type of an empty wedge, i.e., a point, so $\Delta$ is contractible.

In some situations, shellability has stronger topological consequences than homotopy equivalence, as the next result, due to Danaraj and Klee, demonstrates. Say that a pure simplicial complex is thin if every codimension 1 face is contained in exactly two facets, and subthin if it is not thin and every codimension 1 face is contained in at most two facets.

Theorem 3.3.3 ([9]). Let $\Delta$ be a shellable, pure d-dimensional simplicial complex. If $\Delta$ is thin, then $\Delta$ is a PL d-sphere. If $\Delta$ is subthin, then $\Delta$ is a PL d-ball.

\subsubsection{Lexicographic shellability}

In this subsection, we present two conditions on a poset, EL- and CLshellability, that imply shellability of its order complex.

Let $P$ be a finite and bounded poset. An edge-labelling of $P$ is a function $\lambda:\left\{(x, y) \in P^{2} \mid x \triangleleft y\right\} \rightarrow Q$, where $Q$ is some totally ordered set. If $\lambda$ is an edge-labelling of $P$ and $\left\{x_{0} \triangleleft x_{1} \triangleleft \cdots \triangleleft x_{k}\right\}$ is a saturated chain, let $\lambda\left(x_{0}, x_{1}, \ldots, x_{k}\right)=\left(\lambda\left(x_{0}, x_{1}\right), \lambda\left(x_{1}, x_{2}\right), \ldots, \lambda\left(x_{k-1}, x_{k}\right)\right)$. The chain is called increasing if $\lambda\left(x_{i-1}, x_{i}\right) \leq \lambda\left(x_{i}, x_{i+1}\right)$ for all $i \in[k-1]$, and decreasing if $\lambda\left(x_{i-1}, x_{i}\right)>\lambda\left(x_{i}, x_{i+1}\right)$ for all $i \in[k-1]$.

Definition 3.3.4. An edge-labelling $\lambda$ of $P$ is an EL-labelling (short for edgelexicographic labelling) if, for all $x<y$ in $P$, there is exactly one increasing maximal chain in $[x, y]$, say $\left\{x_{0} \triangleleft x_{1} \triangleleft \cdots \triangleleft x_{k}\right\}$, and this chain is lexicographically minimal among the maximal chains in $[x, y]$ (i.e., if $\left\{y_{0} \triangleleft y_{1} \triangleleft\right.$ $\left.\cdots \triangleleft y_{k}\right\}$ is any other maximal chain, then $\lambda\left(x_{j-1}, x_{j}\right)<\lambda\left(y_{j-1}, y_{j}\right)$, where $\left.j=\min \left\{i \in[k] \mid \lambda\left(x_{i-1}, x_{i}\right) \neq \lambda\left(y_{i-1}, y_{i}\right)\right\}\right)$. If $P$ has an EL-labelling, $P$ is called EL-shellable.

The reason for this terminology is the next result, due to Björner [2] in the pure case and to Björner and Wachs [5] in the general case.

Theorem 3.3.5 ([2,5]). Suppose $P$ is a finite and bounded poset with an EL-labelling. Then the lexicographic order of the maximal chains of $P$ is a shelling order of $\Delta(P)$. 


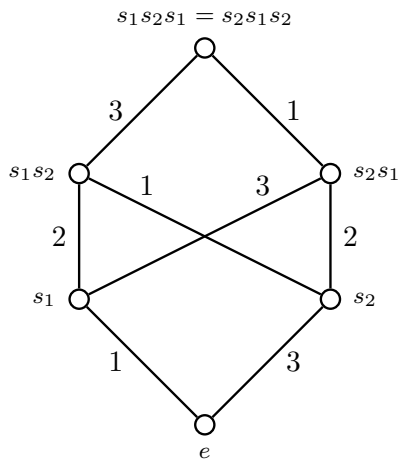

$P=\operatorname{Br}\left(S_{3}\right)$

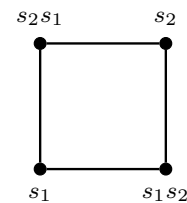

$\Delta(\bar{P})$

Figure 3.3.2: An EL-labelling of $P=\operatorname{Br}\left(S_{3}\right)$ and $\Delta(\bar{P})$.

Clearly, the corresponding order of the maximal chains of $\bar{P}$ is a shelling order of $\Delta(\bar{P})$. It is the topology of the latter order complex which is interesting since $\Delta(P)$ is a cone, hence contractible. Combining Theorems 3.3.2 and 3.3.5 yields the following:

Theorem 3.3.6 ([5]). Let $P$ be a finite and bounded poset with an ELlabelling. Then $\Delta(\bar{P})$ is homotopy equivalent to a wedge of spheres, where for each $d$, the number of $d$-spheres is equal to the number of decreasing maximal $(d+2)$-chains of $P$.

Example 3.3.7. Consider the EL-labelling of $P=\operatorname{Br}\left(S_{3}\right)$ in Figure 3.3.2. It has one decreasing maximal $(d+2)$-chain for $d=1$, and zero for $d \neq 1$. As predicted by Theorem 3.3.6, $\Delta(\bar{P})$ is homotopy equivalent to the 1-sphere.

Let $P$ be a finite and bounded poset. A chain-labelling of $P$ is a function $\lambda:\{\{\hat{0} \triangleleft \cdots \triangleleft x\} \mid x \in P-\{\hat{0}\}\} \rightarrow Q$, where $Q$ is some totally ordered set. If $x<y$ in $P$ and $C$ is a maximal chain in $[\hat{0}, x]$, then for each maximal chain $C^{\prime}=\left\{x_{0} \triangleleft x_{1} \triangleleft \cdots \triangleleft x_{k}\right\}$ in $[x, y]$, let

$$
\lambda_{C}\left(C^{\prime}\right)=\left(\lambda\left(C \cup\left\{x_{1}\right\}\right), \lambda\left(C \cup\left\{x_{1}, x_{2}\right\}\right), \ldots, \lambda\left(C \cup C^{\prime}\right)\right) .
$$

We call $C^{\prime}$ increasing if the sequence $\lambda_{C}\left(C^{\prime}\right)$ is weakly increasing, and $d e$ creasing if $\lambda_{C}\left(C^{\prime}\right)$ is strictly decreasing.

Definition 3.3.8. A chain-labelling $\lambda$ of $P$ is a $C L$-labelling (short for chainlexicographic labelling) if, for all $x<y$ in $P$ and every maximal chain $C$ in $[\hat{0}, x]$, there is exactly one increasing maximal chain $C^{\prime}$ in $[x, y]$ (i.e., $\lambda_{C}\left(C^{\prime}\right)$ 
is weakly increasing), and this chain is lexicographically minimal among the maximal chains in $[x, y]$ (i.e., if $C^{\prime \prime}$ is any other maximal chain, then $\lambda_{C}\left(C^{\prime}\right)$ is lexicographically smaller than $\left.\lambda_{C}\left(C^{\prime \prime}\right)\right)$. If $P$ has a CL-labelling, $P$ is called CL-shellable.

It is clear that every EL-labelling induces a CL-labelling, but unknown whether CL-shellability implies EL-shellability. However, Theorems 3.3.5 and 3.3.6 remain true if one replaces "an EL-labelling" with "a CL-labelling". This was shown by Björner and Wachs in [4] (the pure case) and [5] (the general case). 



\section{Chapter 4}

\section{Combinatorics and topology related to involutions}

\subsection{Topology of Bruhat orders}

\subsubsection{The Bruhat order on parabolic quotients}

Suppose $(W, S)$ is a Coxeter system and $J \subseteq S$. Recall that, in particular, $W^{\emptyset}=W$. An edge-labelling of $\operatorname{Br}\left(W^{J}\right)$ is given by $\lambda(u \triangleleft w)=t$ if $w=t u$, where $T$ is the set of reflections. Edelman [16] defined a total order on $T$ such that this edge-labelling is an EL-labelling for $W=S_{n}$. Proctor [35] extended this result to the classical Weyl groups (i.e., when $W$ is of type $A, B$, or $D$ ). Björner and Wachs [4] proved that the dual of any closed interval in $\operatorname{Br}\left(W^{J}\right)$ is CL-shellable for arbitrary Coxeter systems. (Observe that a poset and its dual contain the same chains, hence have the same order complex.) Dyer [14] later introduced ways to totally order $T$ so that the edge-labelling described above is an EL-labelling of every closed interval in $\operatorname{Br}\left(W^{J}\right)$, this time for any Coxeter system.

Let us describe the chain-labelling of Björner and Wachs. Let $[u, w]$ be an interval in $\operatorname{Br}\left(W^{J}\right)$, and fix a reduced word $s_{1} \cdots s_{k}$ for $w$. It follows from the subword property (Theorem 2.3.2) that if $w \triangleright v \geq u$ in $\operatorname{Br}\left(W^{J}\right)$, then $v=s_{1} \cdots s_{i-1} s_{i+1} \cdots s_{k}$ for some unique $i \in[k]$; let $i$ be the label on the saturated chain $\{w \triangleright v\}$. Similarly, if $v \triangleright v^{\prime} \geq u$, then a reduced word for $v^{\prime}$ is obtained by deleting a unique letter $s_{j}$ in $s_{1} \cdots s_{i-1} s_{i+1} \cdots s_{k}$; let $j$ be the label on the saturated chain $\left\{w \triangleright v \triangleright v^{\prime}\right\}$, and continue until we reach a reduced word for $u$. For example, in the interval $\left[e, s_{1} s_{2} s_{1}\right]$ in $\operatorname{Br}\left(S_{n}\right), n \geq 3$, the maximal chains $\left\{s_{1} s_{2} s_{1} \triangleright s_{1} s_{2} \triangleright s_{1} \triangleright e\right\},\left\{s_{1} s_{2} s_{1} \triangleright s_{1} s_{2} \triangleright s_{2} \triangleright e\right\}$, $\left\{s_{1} s_{2} s_{1} \triangleright s_{2} s_{1} \triangleright s_{1} \triangleright e\right\}$, and $\left\{s_{1} s_{2} s_{1} \triangleright s_{2} s_{1} \triangleright s_{2} \triangleright e\right\}$ receive the labels $(3,2,1),(3,1,2),(1,2,3)$, and $(1,3,2)$, respectively. 
Theorem 4.1.1 ([4]). The chain-labelling described above is a CL-labelling of the dual of an arbitrary closed interval in $\operatorname{Br}\left(W^{J}\right)$. The number of decreasing maximal chains is 1 if $J=\emptyset$ and at most 1 otherwise.

Therefore, by the "CL version" of Theorem 3.3.6, (the order complex of) every open interval $(u, w)$ has the homotopy type of a $(\ell(w)-\ell(u)-2)$-sphere or else is contractible.

However, more can be said. Recall from Subsection 2.3.1 that the Bruhat order on every interval $[u, w]$ in $W^{J}$ is a pure poset whose rank function is given by $\rho(x)=\ell(x)-\ell(u)$. Thus, $\Delta((u, w))$ is pure $(\ell(w)-\ell(u)-2)$ dimensional. Call $[u, w]$ full if it coincides with an interval in $\operatorname{Br}(W)$. It is clear from Theorem 4.1.1 that if $\ell\left(w^{\prime}\right)-\ell\left(u^{\prime}\right)=2$, then $\left|\left[u^{\prime}, w^{\prime}\right]\right| \leq 4$ with equality precisely when $\left[u^{\prime}, w^{\prime}\right]$ is full. Hence, $\Delta((u, w))$ is thin if $[u, w]$ is full and subthin otherwise. From the "CL version" of Theorem 3.3.5 and Theorems 3.3.3 and 4.1.1 we therefore conclude the following.

Theorem 4.1.2 ([4]). Let $[u, w]$ be an interval in $\operatorname{Br}\left(W^{J}\right)$. If $[u, w]$ is full, then $\Delta((u, w))$ is a $P L(\ell(w)-\ell(u)-2)$-sphere. Otherwise, $\Delta((u, w))$ is a $P L(\ell(w)-\ell(u)-2)$-ball.

\subsubsection{The Bruhat order on twisted involutions}

We now return to the set $\mathfrak{I}(\theta)$ of twisted involutions. Recall that

$$
\mathfrak{I}(\theta)=\left\{w \in W \mid \theta(w)=w^{-1}\right\}
$$

where $\theta$ is an involutive automorphism of a Coxeter system $(W, S)$.

For the classical Weyl groups, it can be seen that if $\operatorname{Br}(\mathfrak{I}(\mathrm{id}))$ is pure, then so is $\operatorname{Br}(\mathfrak{I}(\theta))$ for $\theta \neq \mathrm{id}$, and similarly if $\operatorname{Br}(\mathfrak{I}(\mathrm{id}))$ is EL-shellable.

It follows from [38] that $\operatorname{Br}(\mathfrak{I}(\mathrm{id}))$ is a pure poset in type $A$. Incitti [27] reproved this fact using combinatorial methods. He first described the covering relation of $\operatorname{Br}(\mathfrak{I}(\mathrm{id}))$ in terms of the diagrams of the involutions viewed as permutations in $S_{n}$. He then used this description to prove that this is a pure poset whose rank function is given by the average of the number of inversions and the number of exceedances. Recall that a pair $(i, j) \in[n]^{2}$ is an inversion of $\sigma \in S_{n}$ if $i<j$ and $\sigma(i)>\sigma(j)$. An exceedance of $\sigma$ is an element $i \in[n]$ such that $\sigma(i)>i$.

In $[26,28]$, Incitti provided similar characterisations of the covering relation of $\operatorname{Br}(\mathfrak{I}(\mathrm{id}))$ in types $B$ and $D$. He again used these to prove that the induced subposets of the involutions are graded. Moreover, he gave a unified account of the rank function of $\operatorname{Br}(\mathfrak{I}(\mathrm{id}))$ in the classical Weyl groups as the average of the length and the absolute length. As we have seen, this 
was generalised by Hultman [21] to $\operatorname{Br}(\Im(\theta))$ in arbitrary Coxeter groups; see Theorem 2.4.2.

Incitti also used his descriptions of the covering relations to construct EL-labellings of $\operatorname{Br}(\mathfrak{I}(\mathrm{id}))$ in the classical Weyl groups. Recall that part of the definition of an EL-labelling says that in each interval, there is exactly one increasing maximal chain. Incitti proved that in each interval, there is also exactly one decreasing maximal chain. From this it follows (see, e.g., [27, Theorem 2.2]) that $\operatorname{Br}(\mathfrak{I}(\mathrm{id}))$ is eulerian, i.e., each non-trivial closed interval has equally many elements of even rank as elements of odd rank. Theorems 3.3.3 and 3.3.5 thus imply that every open interval in $\operatorname{Br}(\mathfrak{I}(\mathrm{id}))$ is a PL sphere. In [22], Hultman proved that this holds in $\operatorname{Br}(\mathfrak{I}(\theta))$ for any Coxeter system, although it remains an open question whether the spheres are shellable.

\subsubsection{The Bruhat order and zircons}

The concept of a special matching on a poset was introduced by Brenti [6]. For eulerian posets, an equivalent notion was independently introduced by du Cloux [12].

Definition 4.1.3. A special matching on a finite poset $P$ with $\hat{1}$ is a function $M: P \rightarrow P$ such that

- $M^{2}=\mathrm{id}$,

- for all $x \in P$, either $M(x) \triangleleft x$ or $x \triangleleft M(x)$, and

- if $x \triangleleft y$ and $M(x) \neq y$, then $M(x)<M(y)$.

The definition is motivated by the following result, which is a consequence of the lifting property (Proposition 2.3.6).

Proposition 4.1.4. Given a Coxeter system $(W, S)$, let $w \in W$ and suppose $s \in D_{R}(w)$. Then the map $x \mapsto x s$ is a special matching on $[e, w]$.

Definition 4.1.5. A zircon is a poset in which every non-trivial principal order ideal is finite and admits a special matching.

Thus, $\operatorname{Br}(W)$ is always a zircon. The term "zircon" was coined by Marietti [31], although he defined zircons somewhat differently; however, the two definitions are equivalent [23].

A major significance of zircons is captured by the next theorem. It essentially follows from [12], [13], or [37], together with [31]; see [22, Corollary 4.3 and Remark 4.4].

Theorem 4.1.6. The order complex of any open interval in a zircon is a $P L$ sphere. 
It was as a consequence of Theorem 4.1.6 and the following result that Hultman proved that the open intervals in $\operatorname{Br}(\mathfrak{I}(\theta))$ are PL spheres.

Proposition 4.1.7 ([22]). Given a Coxeter system $(W, S)$ with an involutive automorphism $\theta$, let $w \in \mathfrak{I}(\theta)$ and suppose $s \in D_{R}(w)$. Then the map $x \mapsto x \underline{s}$ is a special matching on $[e, w] \subseteq \operatorname{Br}(\mathfrak{I}(\theta))$.

Proposition 4.1.7 can be proved in the same way as Proposition 4.1.4 thanks to the, in many respects, huge resemblance between the combinatorics of $\operatorname{Br}(W)$ and that of $\operatorname{Br}(\mathfrak{I}(\theta))$, in particular, the very similar lifting properties; see Propositions 2.3.6 and 2.4.8.

\subsubsection{The Bruhat order on twisted identities}

Recall that the set of twisted identities is

$$
\iota(\theta)=\left\{\theta\left(w^{-1}\right) w \mid w \in W\right\} \subseteq \mathfrak{I}(\theta) .
$$

The poset $\operatorname{Br}(\iota(\theta))$ is not as well understood as $\operatorname{Br}(\mathfrak{I}(\theta))$. In this subsection, we collect some of the known facts.

Recall that $\operatorname{Br}(\mathfrak{I}(\theta))$ is always graded and that its rank function $\rho$ satisfies $\rho(w)=\left(\ell(w)+\ell^{\theta}(w)\right) / 2=k$, where $k$ is the number of letters in a reduced $\underline{S}$-expression for $w$. Since twisted identities have vanishing twisted absolute length, $\rho(w)=\ell(w) / 2$ if $w \in \iota(\theta)$.

We have the following alternative description of the twisted absolute length $\ell^{\theta}$; see [24], where it is also shown that this makes $\ell^{\theta}$ well defined.

Definition 4.1.8. Let $\underline{s}_{1} \cdots \underline{s}_{k}$ be a reduced $\underline{S}$-expression for $w \in \mathfrak{I}(\theta)$. Then the twisted absolute length of $w$, denoted $\ell^{\theta}(w)$, is the number of indices $i \in[k]$ such that $\underline{s}_{1} \cdots \underline{s}_{i-1} \underline{s}_{i}=\underline{s}_{1} \cdots \underline{s}_{i-1} s_{i}$.

It follows from Definition 4.1.8 that whenever $\operatorname{Br}(\iota(\theta))$ is graded, its rank function is $\rho$. Indeed, if $w=\underline{s}_{1} \cdots \underline{s}_{k} \in \iota(\theta)$ with $\rho(w)=k$, then $e \triangleleft \underline{s}_{1} \triangleleft$ $\underline{s}_{1} \underline{s}_{2} \triangleleft \cdots \triangleleft w$ in $\operatorname{Br}(\iota(\theta))$.

Unlike $\operatorname{Br}(\mathfrak{I}(\theta)), \operatorname{Br}(\iota(\theta))$ is not always graded:

Example 4.1.9 ([24]). Suppose $S=\left\{s_{1}, s_{2}, s_{3}\right\}$ with $s_{1} s_{2}, s_{1} s_{3}$, and $s_{2} s_{3}$ of order 3 (in other words, $W$ is the affine Weyl group $\widetilde{A}_{2}$ corresponding to $A_{2}$ ), and let $\theta\left(s_{1}\right)=s_{1}, \theta\left(s_{2}\right)=s_{3}$, and $\theta\left(s_{3}\right)=s_{2}$. Then $e \triangleleft \underline{s}_{3} \triangleleft \underline{s}_{2} \underline{s}_{1} \underline{s}_{3}$ and $e \triangleleft \underline{s}_{2} \triangleleft \underline{s}_{2} \underline{s}_{1} \triangleleft \underline{s}_{2} \underline{s}_{1} \underline{s}_{3}$ in $\operatorname{Br}(\iota(\theta))$; see Figure 4.1.1.

However, $\operatorname{Br}(\iota(\theta))$ is often graded, as we shall now see. Say that $\theta$ has the no odd flip, or NOF, property if the order of $s \theta(s)$ is even or infinite for all $s \in S$ with $s \neq \theta(s)$. This means that $\theta$ never flips an edge with an odd label in the Coxeter graph. 


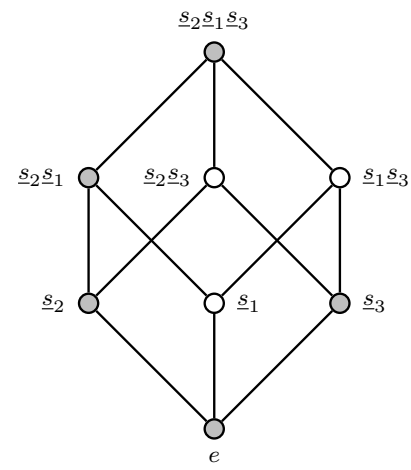

Figure 4.1.1: The interval $\left[e, \underline{s}_{2} \underline{s}_{1} \underline{s}_{3}\right] \subseteq \operatorname{Br}(\Im(\theta))$, where $\theta$ is the non-trivial involution of $\widetilde{A}_{2}$ which fixes $s_{1}$. If a twisted involution is marked by a $\bigcirc$, it belongs to $\iota(\theta)$; if it is marked by a $\mathrm{O}$, it does not.

Theorem 4.1.10 ([24]). Assume that $\theta$ has the NOF property. Then $\operatorname{Br}(\iota(\theta))$ is graded with rank function $\rho$, and if $(W, S)$ is finite and irreducible, then $\operatorname{Br}(\iota(\theta))$ is bounded and pure.

If $(W, S)$ is finite and irreducible, then $\theta$ has the NOF property, unless the type is $A_{2 n} \cong S_{2 n+1}$ or $I_{2}(2 n+1)$ for some $n \geq 1$, and $\theta$ is the unique non-trivial automorphism. In the latter case, however, it is easy to see that $\operatorname{Br}(\iota(\theta))$ is pure.

Conjecture 4.1.11 ([24]). If $W=A_{2 n}$, then $\operatorname{Br}(\iota(\theta))$ is pure.

If $\operatorname{Br}(W)$ is finite, it contains a maximum $w_{0}$, and $\theta(w)=w_{0} w w_{0}$ defines an involutive automorphism of $(W, S)$. Since $\iota(\theta)=\left\{w_{0} w^{-1} w_{0} w \mid w \in W\right\}$ we have

$$
w_{0} \cdot \iota(\theta)=\left\{w^{-1} w_{0} w \mid w \in W\right\} .
$$

Since left (as well as right) multiplication by $w_{0}$ is an anti-automorphism of $\operatorname{Br}(W), \operatorname{Br}(\iota(\theta))$ is isomorphic to the dual of $\operatorname{Br}\left(\left[w_{0}\right]\right)$, where $\left[w_{0}\right]$ is the conjugacy class of $w_{0}$.

When $W=S_{n}$, this $\theta$ is the unique non-trivial automorphism of $(W, S)$, mapping $s_{i}=(i, i+1)$ to $s_{n-i}$. For even $n,\left[w_{0}\right]$ is the set of fixed-point-free involutions. For odd $n,\left[w_{0}\right]$ is the set of involutions with exactly one fixed point. Thus, Conjecture 4.1.11 can be rephrased as follows:

Conjecture 4.1.12 ([24]). The subposet of $\operatorname{Br}\left(S_{2 n+1}\right)$ induced by the involutions with exactly one fixed point is pure. 
Let us now consider the topology of intervals in $\operatorname{Br}(\iota(\theta))$, under the NOF assumption. Call an interval $[u, w] \subseteq \operatorname{Br}(\iota(\theta))$ full if it coincides with an interval in $\operatorname{Br}(\mathfrak{I}(\theta))$. Thus, $\Delta((u, w))$ is a $\mathrm{PL}$ sphere if $[u, w]$ is full. This characterises full intervals in $\operatorname{Br}(\iota(\theta))$ :

Theorem 4.1.13 ([24]). Assume that $\theta$ has the NOF property. Consider an interval $[u, w] \subseteq \operatorname{Br}(\iota(\theta))$. If it is full, then $\Delta((u, w))$ is a $P L(\rho(w)-\rho(u)-2)$ sphere. Otherwise, $\Delta((u, w))$ is $\mathbf{Z}$-acyclic (i.e., has trivial reduced integral homology).

Conjecture 4.1.14 ([24]). Assume that $\theta$ has the NOF property. Consider an interval $[u, w] \subseteq \operatorname{Br}(\iota(\theta))$. If it is not full, then $\Delta((u, w))$ is a PL ball of dimension $\rho(w)-\rho(u)-2$.

A few years ago, an important special case of Conjecture 4.1.14 was proved by Can, Cherniavsky, and Twelbeck by establishing shellability of the intervals.

Theorem 4.1.15 ([7]). If $W=A_{2 n-1}$, then $\operatorname{Br}(\iota(\theta))$ is EL-shellable.

More precisely, they constructed an EL-labelling of the subposet $\operatorname{Br}\left(F_{2 n}^{0}\right)$ of $\operatorname{Br}\left(S_{2 n}\right)$ induced by the fixed-point-free involutions, relying heavily on Incitti's characterisation of the covering relation of $\operatorname{Br}(\mathfrak{I}(\mathrm{id}))$ in type $A$.

Because $\operatorname{Br}\left(F_{2 n}^{0}\right)$ is a pure, proper, full rank subposet of an interval in $\operatorname{Br}(\mathfrak{I}(\mathrm{id}))$, and the latter poset is eulerian [27], Theorem 4.1.15 together with Theorems 3.3.3 and 3.3.5 yield the following result, where the dimension can be computed from the fact that the rank function of $\operatorname{Br}(\Im(i d))$ is given by the average of the inversion number and the exceedance number.

Theorem 4.1.16 ([7]). The order complex of the proper part of $\operatorname{Br}\left(F_{2 n}^{0}\right)$ is a PL ball of dimension $n^{2}-n-2$.

\subsection{Word properties for twisted involutions}

Recall from Subsection 2.4.1 that twisted involutions can be represented by words in the alphabet $\underline{S}=\{\underline{s} \mid s \in S\}$, called $\underline{S}$-expressions. An $\underline{S}$ expression representing $w \in \mathfrak{I}(\theta)$ is called a reduced $\underline{S}$-expression for $w$ if it is shortest possible. In analogy with the situation for words in the alphabet $S$, if $s s^{\prime}$ has finite order $m\left(s, s^{\prime}\right)$, let a braid move be the replacement of $\underline{s} \underline{s}^{\prime} \underline{s} \cdots$ by $\underline{s}^{\prime} \underline{s} \underline{s}^{\prime} \cdots$, both consisting of $m\left(s, s^{\prime}\right)$ letters. It is known that braid moves in a reduced $\underline{S}$-expression preserve the twisted involution (it follows from [38] when $W$ is a Weyl group, and from [17] in the general case). However, they do not in general suffice to connect all the reduced $\underline{S}$-expressions, as the next example shows. 
Example 4.2.1. Let $W=S_{4}, s_{i}=(i, i+1)$, and $\theta=\mathrm{id}$. Then $\underline{s}_{3} \underline{s}_{2} \underline{s}_{1} \underline{s}_{2}$ and $\underline{s}_{2} \underline{s}_{3} \underline{s}_{1} \underline{s}_{2}$ represent the same (ordinary) involution, namely the longest group element, but they cannot be connected using only braid moves.

Therefore, other moves must be added to the braid moves, in order to connect all reduced $\underline{S}$-expressions for any given $w \in \mathfrak{I}(\theta)$. Since one of the main motivations is to solve the word problem for $\mathfrak{I}(\theta)$, i.e., the problem of deciding whether two $\underline{S}$-expressions represent the same twisted involution, these additional moves have to preserve $w$. Ideally, in the search for word properties for $\mathfrak{I}(\theta)$ analogous to Theorem 2.2.4, one would like to find a minimal set of additional moves which is easy to determine from the Coxeter graph of $(W, S)$.

Denote by $\hat{\mathcal{R}}_{\theta}(w)$ the set of all reduced $\underline{S}$-expressions for $w \in \mathfrak{I}(\theta)$. An initial move is the replacement of one element in $\hat{\mathcal{R}}_{\theta}(v)$ (for some $v \in \mathfrak{I}(\theta)$ ) by another, in the beginning of a reduced $\underline{S}$-expression for some $w \in \mathfrak{I}(\theta)$. In particular, let a half-braid move be the the replacement of $\underline{s} \underline{s}^{\prime}$ by $\underline{s}^{\prime} \underline{s}$ (or $\underline{s} \underline{s}^{\prime} \underline{s}$ by $\left.\underline{s}^{\prime} \underline{s} \underline{s}^{\prime}\right)$ if these are the first letters in a reduced $\underline{S}$-expression, and $m\left(s, s^{\prime}\right)=3\left(m\left(s, s^{\prime}\right)=4\right)$. A few years ago, $\mathrm{Hu}$ and Zhang proved a word property for $\mathfrak{I}(\mathrm{id})$ in the case of the classical Weyl groups.

Theorem 4.2.2 ([18, 19]). Let $(W, S)$ be a Coxeter system and let $w \in \mathfrak{I}(\mathrm{id})$, and suppose the generator indexing is as in Table 2.1 at the end of Chapter 2.

(i) In type $A_{n}$, braid moves and half-braid moves suffice to connect $\hat{\mathcal{R}}_{\mathrm{id}}(w)$.

(ii) In type $B_{n}$, braid moves, half-braid moves, and the initial move

$$
\underline{s}_{1} \underline{s}_{2} \underline{s}_{3} \underline{s}_{1} \underline{s}_{2} \underline{s}_{1} \longleftrightarrow \underline{s}_{1} \underline{s}_{2} \underline{s}_{3} \underline{s}_{2} \underline{s}_{1} \underline{s}_{2}
$$

suffice to connect $\hat{\mathcal{R}}_{\mathrm{id}}(w)$.

(iii) In type $D_{n}$, braid moves, half-braid moves, and the initial move

$$
\underline{s}_{4} \underline{s}_{2} \underline{s}_{1} \underline{s}_{3} \underline{s}_{2} \underline{s}_{1} \underline{s}_{3} \underline{s}_{4} \longleftrightarrow \underline{s}_{4} \underline{s}_{2} \underline{s}_{1} \underline{s}_{3} \underline{s}_{2} \underline{s}_{1} \underline{s}_{4} \underline{s}_{3}
$$

suffice to connect $\hat{\mathcal{R}}_{\mathrm{id}}(w)$.

Remark. Although not mentioned by $\mathrm{Hu}$ and Zhang, it should be observed that the involutions $\underline{s}_{1} \underline{s}_{2} \underline{s}_{3} \underline{s}_{1} \underline{s}_{2} \underline{s}_{1}$ and $\underline{s}_{4} \underline{s}_{2} \underline{s}_{1} \underline{s}_{3} \underline{s}_{2} \underline{s}_{1} \underline{s}_{3} \underline{s}_{4}$ are the longest elements in the parabolic subgroups of types $B_{3}$ and $D_{4}$, respectively.

Example 4.2.3. Let $W=S_{4}, s_{i}=(i, i+1)$, and $\theta=\mathrm{id}$. Then $\underline{s}_{1} \underline{s}_{3} \underline{s}_{2} \underline{s}_{1}$ and $\underline{s}_{2} \underline{s}_{3} \underline{s}_{1} \underline{s}_{2}$ both represent the involution 4321 (in one-line notation) and they can be connected by the following sequence of braid moves and half-braid moves:

$$
\underline{s}_{1} \underline{s}_{3} \underline{s}_{2} \underline{s}_{1}=\underline{s}_{3} \underline{s}_{1} \underline{s}_{2} \underline{s}_{1}=\underline{s}_{3} \underline{s}_{2} \underline{s}_{1} \underline{s}_{2}=\underline{s}_{2} \underline{s}_{3} \underline{s}_{1} \underline{s}_{2}
$$


Here $\underline{s}_{1} \underline{s}_{3} \leftrightarrow \underline{s}_{3} s_{1}$ and $\underline{s}_{1} \underline{s}_{2} \underline{s}_{1} \leftrightarrow \underline{s}_{2} \underline{s}_{1} \underline{s}_{2}$ are braid moves, while $\underline{s}_{3} \underline{s}_{2} \leftrightarrow \underline{s}_{2} \underline{s}_{3}$ is a half-braid move. Observe that the last move is where we got stuck in Example 4.2.1. Also note that a "half-braid move" which is not performed as an initial move will in general change the group element. For instance,

$$
\underline{s}_{1} \underline{s}_{3} \underline{s}_{2} \underline{s}_{1} \neq \underline{s}_{1} \underline{s}_{3} \underline{s}_{1} \underline{s}_{2}=\underline{s}_{3} \underline{s}_{1} \underline{s}_{1} \underline{s}_{2}=\underline{s}_{3} \underline{s}_{2}
$$

In [17], Hamaker, Marberg, and Pawlowski provided, for any $(W, S)$ and any $\theta$, a set of moves that can be added to the braid moves, in order to connect $\hat{\mathcal{R}}_{\theta}(w)$. However, it is not a minimal set of moves, nor is it easy to determine from the Coxeter graph of $(W, S)$. In particular, their word property reduces to weaker statements than $\mathrm{Hu}$ and Zhang's results in those special cases.

Recently, Marberg [30] identified a minimal set of additional moves, easily determined from the Coxeter graph, for all finite and affine types with arbitrary $\theta$. His word property does reduce to Theorem 4.2.2. It is interesting to note that the non-braid moves needed in the finite types suffice also in the affine types. For a statement of this word property, see Theorem 5.2.1 confined to the case when $(W, S)$ is finite or affine. ${ }^{1}$

\footnotetext{
${ }^{1}$ Marberg's word property only has one initial move of each type; see the discussion in Section 5.2.
} 


\section{Chapter 5}

\section{Summary of papers}

\subsection{Summary of Paper A}

We study conjugacy classes, and more generally, unions of conjugacy classes, of involutions in the symmetric group $S_{n}$. Since two permutations are conjugate if and only if, for each $i \geq 1$, they have the same number of disjoint cycles of length $i$, two involutions in $S_{n}$ are conjugate precisely when they have the same number of fixed points. Denote by $F_{n}^{a}$ the conjugacy class in $S_{n}$ consisting of the involutions with exactly $a$ fixed points, where $a \in\{0,1, \ldots, n\}$. Observe that $F_{n}^{0}$ is the set of fixed-point-free involutions, while $F_{n}^{1}$ is the set of involutions with exactly one fixed point. More generally, for $A \subseteq\{0,1, \ldots, n\}$, let

$$
F_{n}^{A}=\bigcup_{a \in A} F_{n}^{a} .
$$

We regard $F_{n}^{A}$ as the poset $\operatorname{Br}\left(F_{n}^{A}\right)$, i.e., with the order induced by the Bruhat order on $S_{n}$. Therefore, $\operatorname{Br}\left(F_{n}^{0}\right)$ and $\operatorname{Br}\left(F_{n}^{1}\right)$ are isomorphic to the duals of $\operatorname{Br}(\iota(\theta))$ with $\theta \neq$ id in type $A_{n-1}$ for $n$ even and for $n$ odd, respectively.

Our main result is a complete classification of the sets $A$ for which $\operatorname{Br}\left(F_{n}^{A}\right)$ is pure. Informally, $\operatorname{Br}\left(F_{n}^{A}\right)$ is pure precisely when $A-\{n\}$ is empty or an "interval", which may consist of a single element if it is 0,1 , or $n-2$. It follows from the main result that $\operatorname{Br}\left(F_{n}^{0}\right), \operatorname{Br}\left(F_{n}^{1}\right), \operatorname{Br}\left(F_{n}^{n-2}\right)$, and $\operatorname{Br}\left(F_{n}^{n}\right)$ are the only pure conjugacy classes of involutions in $S_{n}$. The fact that $\operatorname{Br}\left(F_{n}^{1}\right)$ is pure gives an affirmative answer to Conjecture 4.1.12. Recall from Subsection 4.1.4 that this means that $\operatorname{Br}(\iota(\theta))$ is pure if $(W, S)$ is finite and irreducible. From this it follows that, in fact, $\operatorname{Br}(\iota(\theta))$ is pure whenever $W$ is finite.

Put $I_{n}=\mathfrak{I}(\mathrm{id})$, the set of involutions in $S_{n}$. The proof of the main result fundamentally relies on Incitti's characterisation of the covering relation of $\operatorname{Br}\left(I_{n}\right)$. We also make heavy use of the fact, shown by Incitti, that there 
exists an edge-labelling of $\operatorname{Br}\left(I_{n}\right)$ such that each interval contains a unique increasing maximal chain and a unique decreasing maximal chain. As steps in the proof, we show that $\operatorname{Br}\left(F_{n}^{A}\right)$ always has a maximum and that all its minimal elements have the same rank in $\operatorname{Br}\left(I_{n}\right)$. We then prove that for certain $A$, if $\sigma \triangleleft \tau$ in $\operatorname{Br}\left(F_{n}^{A}\right)$, then $\sigma \triangleleft \tau$ in $\operatorname{Br}\left(I_{n}\right)$. Thus, since $\operatorname{Br}\left(I_{n}\right)$ is pure, so is $\operatorname{Br}\left(F_{n}^{A}\right)$. In these cases, we give the rank function of $\operatorname{Br}\left(F_{n}^{A}\right)$. It is very similar to the rank function of $I_{n}$ (i.e., the average of the inversion number and the exceedance number), which is explained by the fact that $\sigma \triangleleft \tau$ in $\operatorname{Br}\left(F_{n}^{A}\right)$ implies $\sigma \triangleleft \tau$ in $\operatorname{Br}\left(I_{n}\right)$. For the other $A$, we establish impurity by constructing two maximal chains of different lengths.

The paper ends with a short, new proof of the EL-shellability of $\operatorname{Br}\left(F_{n}^{0}\right)$, proved by Can, Cherniavsky, and Twelbeck (see Theorem 4.1.15).

\subsection{Summary of Paper B}

We prove a word property for the set $\mathfrak{I}(\theta)=\left\{w \in W \mid \theta(w)=w^{-1}\right\}$ of twisted involutions, for any Coxeter system $(W, S)$ and any involutive automorphism $\theta$. More precisely, we provide a minimal set of moves, easily determined from the Coxeter graph of $(W, S)$, that can be added to the braid moves in order to connect all reduced $\underline{S}$-expressions for any given $w \in$ $\mathfrak{I}(\theta)$. This generalises the results of $\mathrm{Hu}$ and Zhang [18, 19] and Marberg [30] described in Section 4.2. Recall that the non-braid moves needed in the finite types suffice also in the affine types. It turns out that, in fact, they suffice in all Coxeter groups.

We need some notation in order to state our word property. Recall that an initial move is the replacement of one element in $\hat{\mathcal{R}}_{\theta}(v)$ (for some $v \in \mathfrak{I}(\theta)$ ) by another, in the beginning of a reduced $\underline{S}$-expression for some $w \in \mathfrak{I}(\theta)$. If $J \subseteq S$ generates a finite parabolic subgroup $W_{J}$ of $W$, let $w_{0}(J)$ denote its longest element. When $\theta(J)=J, \theta_{J}$ is the restriction of $\theta$ to $W_{J}$.

Theorem 5.2.1. Let $(W, S)$ be a Coxeter system with an involutive automorphism $\theta$, and let $w \in \mathfrak{I}(\theta)$. Then any two reduced $\underline{S}$-expressions for $w$ can be connected by a sequence of braid moves and initial moves that replace $x$ with $y$ if $x, y \in \hat{\mathcal{R}}_{\theta}\left(w_{0}(J)\right)$ for some $\theta$-stable $J \subseteq S$. The following $W_{J}$ and $\theta_{J}$ are necessary and sufficient:

- $W_{J}$ of type $A_{3}$ with $\theta_{J} \neq \mathrm{id}$;

- $W_{J}$ of type $B_{3}$;

- $W_{J}$ of type $D_{4}$ with $\theta_{J}=\mathrm{id}$;

- $W_{J}$ of type $\mathrm{H}_{3}$;

- $W_{J}$ of type $I_{2}(m), 3 \leq m<\infty$, with $\theta_{J}=\mathrm{id}$;

- $W_{J}$ of type $I_{2}(m), 2 \leq m<\infty$, with $\theta_{J} \neq \mathrm{id}$. 

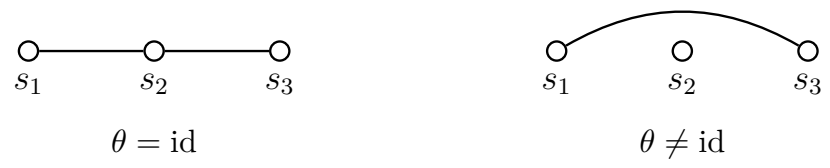

Figure 5.2.1: The graph $G(w, \theta)$, where $w$ is the longest element in $S_{4}$ (as always, $\left.s_{i}=(i, i+1)\right)$. Observe that $G(w, \theta)$ is connected when $\theta=\mathrm{id}$, but disconnected when $\theta \neq$ id.

Some observations are in order. First, the listed $W_{J}$ (except the reducible dihedral group $\left.I_{2}(2)\right)$ are precisely the finite types for which the complement of the Coxeter graph is disconnected. Second, when $\theta_{J}=\mathrm{id}$, the $I_{2}(3)$ and $I_{2}(4)$ initial moves are the half-braid moves in Theorem 4.2.2. Finally, note that $I_{2}(2)$ does not appear in the list when $\theta_{J}=\mathrm{id}$. The corresponding move is allowed, but it is a braid move in this case.

In order to prove Theorem 5.2.1, it is crucial to understand when there exist reduced $\underline{S}$-expressions for $w \in \mathfrak{I}(\theta)$ that end with the longest possible alternating sequence of two given right descents.

Definition 5.2.2. Given $w \in \mathfrak{I}(\theta)$ and $s, s^{\prime} \in D_{R}(w), s \neq s^{\prime}$, say that $w$ is $\left(s, s^{\prime}\right)$-maximal if it has a reduced $\underline{S}$-expression of the form

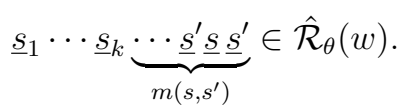

Since braid moves in a reduced $\underline{S}$-expression preserve the twisted involution, any $\left(s, s^{\prime}\right)$-maximal element is also $\left(s^{\prime}, s\right)$-maximal. The main technical ingredient in the proof of Theorem 5.2.1 is a characterisation of the $\left(s, s^{\prime}\right)$ maximal elements.

It is convenient to encode the information conveyed by this characterisation in a graph. To this end, let $G(w, \theta)$ be the graph on vertex set $D_{R}(w)$ in which $\left\{s, s^{\prime}\right\}$ is an edge if and only if $w$ is $\left(s, s^{\prime}\right)$-maximal. See Figure 5.2.1 for an example.

Let us describe the basic strategy employed in the proof of Theorem 5.2.1. Starting with a list composed of all braid moves, we work by induction on the rank of a given twisted involution $w$. If the moves that are so far collected do not suffice to connect all the reduced $\underline{S}$-expressions for $w$, sufficient new ones are added to the list. The proof shows that new moves must be added precisely when $G(w, \theta)$ is disconnected. We use the characterisation of $\left(s, s^{\prime}\right)$ maximal elements to prove that this can only happen when $w$ is the longest element of a finite, $\theta$-stable parabolic subgroup $W_{J}$. Another application of 
this characterisation, together with the classification of finite Coxeter groups, yields the list of such $W_{J}$; this list is precisely the one given in Theorem 5.2.1.

Theorem 5.2.1 can be sharpened in the following sense: only one initial move of each type is needed, provided $x$ and $y$ are appropriately chosen. This is because when $G\left(w_{0}(J), \theta\right)$ is disconnected, it has exactly two connected components, and it follows from the proof of Theorem 5.2.1 that it suffices to allow the replacement of one fixed prefix whose last letter is in one component of $G\left(w_{0}(J), \theta\right)$, by one whose last letter is in the other component. We provide a list of such $x$ and $y$. This makes it clear how Theorem 5.2.1 generalises Theorem 4.2.2 and Marberg's word property.

The paper ends with consequences of Theorem 5.2.1 in some special cases, e.g., in the context of right-angled Coxeter systems.

Let us describe two corollaries of Theorem 5.2.1 which are not present in Paper B.

It follows from Definition 4.1.8 that a twisted involution $w$ is a twisted identity if and only if, for some reduced $\underline{S}$-expression $\underline{s}_{1} \cdots \underline{s}_{k}$ for $w$ and each $i \in[k], \underline{s}_{1} \cdots \underline{s}_{i-1} \underline{s}_{i} \neq \underline{s}_{1} \cdots \underline{s}_{i-1} s_{i}$. It can be checked that of the $W_{J}$ listed in Theorem 5.2.1, only type $I_{2}(m)$ with $m$ even and $\theta_{J} \neq \mathrm{id}$, has a longest element which is a twisted identity. Therefore, Theorem 5.2.1 implies the following result.

Corollary 5.2.3. Let $(W, S)$ be a Coxeter system with an involutive automorphism $\theta$, and let $w \in \iota(\theta)$. Then any two reduced $\underline{S}$-expressions for $w$ can be connected by a sequence of braid moves and initial moves that replace $x$ with $y$ if $x, y \in \hat{\mathcal{R}}_{\theta}\left(w_{0}(J)\right)$ for some $\theta$-stable $J \subseteq S$, where $W_{J}$ is of type $I_{2}(m)$ with $m$ even and $\theta_{J} \neq \mathrm{id}$.

Recall from Corollary 2.2.6 that any expression (not necessarily reduced) for $w$ can be transformed into any reduced expression for $w$ by a sequence of braid moves and deletions of factors $s s$ for $s \in S$. We shall prove an analogous result for $\mathfrak{I}(\theta)$. Some caution is required, because braid moves in an unreduced $\underline{S}$-expression need not preserve the twisted involution. For instance, consider $\underline{s}_{1} \underline{s}_{2} \underline{s}_{1}$ and $\underline{s}_{2} \underline{s}_{1} \underline{s}_{2}$ in $S_{3}$ with $\theta=$ id. Then $\underline{s}_{1} \underline{s}_{2} \underline{s}_{1} \leftrightarrow \underline{s}_{2} \underline{s}_{1} \underline{s}_{2}$ is a braid move, but $\underline{s}_{1} \underline{s}_{2} \underline{s}_{1}=\underline{s}_{2} \underline{s}_{1} \underline{s}_{1}=\underline{s}_{2}=s_{2} \neq s_{1}=\underline{s}_{1}=\underline{s}_{1} \underline{s}_{2} \underline{s}_{2}=\underline{s}_{2} \underline{s}_{1} \underline{s}_{2}$.

Given an $\underline{S}$-expression $\underline{s}_{1} \cdots \underline{s}_{k}$, call a braid move in $\underline{s}_{1} \cdots \underline{s}_{k}$ reduced if it is a braid move in $\underline{s}_{1} \cdots \underline{s}_{i}$ for some reduced $\underline{S}$-expression $\underline{s}_{1} \cdots \underline{s}_{i}, i \in[k]$. It is clear that reduced braid moves in an $\underline{S}$-expression preserve the twisted involution.

We can now prove an analogue of Corollary 2.2.6, with a proof similar to that of [3, Theorem 3.3.1(i)]. 
Corollary 5.2.4. Let $(W, S)$ be a Coxeter system with an involutive automorphism $\theta$, and let $w \in \mathfrak{I}(\theta)$. Then any $\underline{S}$-expression $\underline{s}_{1} \cdots \underline{s}_{k}$ for $w$ can be transformed into any reduced $\underline{S}$-expression for $w$ via a sequence of reduced braid moves, initial moves of the kinds listed in Theorem 5.2.1, and deletions of factors $\underline{s} \underline{s}$ for $\underline{s} \in \underline{S}$.

Proof. We use induction on $k$. By Theorem 5.2.1, the result holds if $k=\rho(w)$, so assume that $k>\rho(w)$. Let $j$ be maximal such that $\underline{s}_{1} \cdots \underline{s}_{j}$ is reduced. Then, by the exchange property for $\mathfrak{I}(\theta)$ (Proposition 2.4.6), $\underline{s}_{1} \cdots \underline{s}_{j}=$ $\underline{s}_{1} \cdots \underline{s}_{i-1} \underline{s}_{i+1} \cdots \underline{s}_{j} \underline{s}_{j+1}$ for some $i \in[j]$. By Theorem 5.2.1, these two reduced $\underline{S}$-expressions can be connected by some sequence of braid moves and initial moves of the listed kinds. Moreover,

$$
\begin{aligned}
\left(\underline{s}_{1} \cdots \underline{s}_{j}\right) \underline{s}_{j+1} \underline{s}_{j+2} \cdots \underline{s}_{k} & =\left(\underline{s}_{1} \cdots \underline{s}_{i-1} \underline{s}_{i+1} \cdots \underline{s}_{j} \underline{s}_{j+1}\right) \underline{s}_{j+1} \underline{s}_{j+2} \cdots \underline{s}_{k} \\
& =\underline{s}_{1} \cdots \underline{s}_{i-1} \underline{s}_{i+1} \cdots \underline{s}_{j} \underline{s}_{j+2} \cdots \underline{s}_{k} .
\end{aligned}
$$

Therefore, $\underline{s}_{1} \cdots \underline{s}_{k}$ can be transformed into $\underline{s}_{1} \cdots \underline{s}_{i-1} \underline{s}_{i+1} \cdots \underline{s}_{j} \underline{s}_{j+2} \cdots \underline{s}_{k}$ by the same sequence, followed by a deletion of the factor $\underline{s}_{j+1} \underline{s}_{j+1}$. The result follows by induction.

Consequently, the word problem for $\underline{S}^{*}$ has the following solution. Given an $\underline{S}$-expression $\alpha \in \underline{S}^{*}$, it follows from Corollary 5.2 .4 that $\hat{\mathcal{R}}_{\theta}(\alpha)$ is equal to the set of all reduced $\underline{S}$-expressions which can be obtained from $\alpha$ by some sequence of reduced braid moves, initial moves of the kinds listed in Theorem 5.2.1, and deletions of factors $\underline{s} \underline{s}$. Hence, $\hat{\mathcal{R}}_{\theta}(\alpha)$ can be computed as follows. Suppose $\alpha=\underline{s}_{1} \cdots \underline{s}_{k}$. Observe that if $\underline{s}_{1} \cdots \underline{s}_{j}$ is reduced, then $\underline{s}_{1} \cdots \underline{s}_{j+1}$ is unreduced if and only if some reduced $\underline{S}$-expression for $\underline{s}_{1} \cdots \underline{s}_{j}$ ends with $\underline{s}_{j+1}$, which can be checked using Theorem 5.2.1. Thus, one can obtain a reduced $\underline{S}$-expression for $\underline{s}_{1} \cdots \underline{s}_{k}$ as in the proof of Corollary 5.2.4, and Theorem 5.2.1 can then be used to list all of them.

\subsection{Summary of Paper C}

In [1], Abdallah and Hultman generalised the notion of a special matching on a poset as follows.

Definition 5.3.1. A special partial matching, or $S P M$, on a finite poset $P$ with $\hat{1}$ is a function $M: P \rightarrow P$ such that

- $M^{2}=\mathrm{id}$,

- $M(\hat{1}) \triangleleft \hat{1}$,

- for all $x \in P$, we have $M(x) \triangleleft x, M(x)=x$, or $x \triangleleft M(x)$, and

- if $x \triangleleft y$ and $M(x) \neq y$, then $M(x)<M(y)$. 
Observe that an SPM without fixed points is precisely a special matching; see Definition 4.1.3.

We study the topology of posets with SPMs. Our first main result is the following theorem. It essentially provides a way in which the PL ball or sphere property can be "lifted" from a subinterval.

Theorem 5.3.2. Let $P$ be a finite poset with $\hat{0}$ and $\hat{1}$, and suppose $M$ is an $S P M$ on $P$. If $\Delta((\hat{0}, M(\hat{1})))$ is a PL d-ball, then $\Delta(\bar{P})$ is a $P L(d+1)$-ball. If $\Delta((\hat{0}, M(\hat{1})))$ is a $P L d$-sphere, then $\Delta(\bar{P})$ is a $P L(d+1)$-ball or sphere; the latter holds if and only if $M$ is actually a special matching.

We generalise the concept of a zircon (Definition 4.1.5) to the SPM setting in the obvious way:

Definition 5.3.3. A pircon is a poset in which every non-trivial principal order ideal is finite and admits an SPM.

Clearly, zircons are pircons. Recall Theorem 4.1.6: every open interval in a zircon is a PL sphere. Theorem 5.3.2 is important because it enables us to generalise Theorem 4.1.6 to pircons, which is our second main result.

Theorem 5.3.4. The order complex of any open interval in a pircon is a $P L$ ball or a PL sphere. Moreover, all open intervals are spheres precisely when $P$ is a zircon.

In [37], Reading gave a proof of Theorem 4.1.6. The basic strategy that we follow in the proof of Theorem 5.3.2 is inspired by that proof. Assume $P$ is a finite poset with $\hat{0}, \hat{1}$, and an $\mathrm{SPM} M$, and let $Q=[\hat{0}, M(\hat{1})] \times \mathbf{2}$, where 2 is a totally ordered, two-element set. It turns out that if $\Delta((\hat{0}, M(\hat{1})))$ is a PL $d$-ball (sphere), then $\Delta(\bar{Q})$ is a PL $(d+1)$-ball (sphere). Furthermore, we demonstrate that (a poset isomorphic to) $P$ can be obtained from $Q$ using certain modifications, which we call clean zippings and removals.

Reading proved that clean zippings preserve PL spheres, i.e., if $R^{\prime}$ is obtained from $R$ by a clean zipping and $\Delta(\bar{R})$ is a PL sphere, then $\Delta\left(\overline{R^{\prime}}\right)$ is a PL sphere of the same dimension. We prove that clean zippings preserve PL balls, too. The fact that balls, unlike spheres, have boundaries causes complications.

The main idea is to obtain $\Delta\left(\overline{R^{\prime}}\right)$ from $\Delta(\bar{R})$ by removing a PL ball and inserting another one, in a way which leaves the topology unchanged. We show that this is indeed the case under certain conditions involving the boundaries. These conditions are shown to be satisfied in the situations that appear in our proof. Using similar ideas, a less involved argument proves 
that removals turn both PL balls and PL spheres into PL balls of the same dimension.

Recall from Subsection 4.1.3 that Hultman proved that the open intervals in $\operatorname{Br}(\mathfrak{I}(\theta))$ are PL spheres by showing that $\operatorname{Br}(\mathfrak{I}(\theta))$ is a zircon. Similarly, we prove that under the NOF assumption, the open intervals in $\operatorname{Br}(\iota(\theta))$ are PL balls or PL spheres by showing that $\operatorname{Br}(\iota(\theta))$ is a pircon. This confirms Conjecture 4.1.14.

Without the NOF property, $\operatorname{Br}(\iota(\theta))$ is in general not a pircon. We demonstrate that $\operatorname{Br}(\iota(\theta))$ is not a pircon in type $A_{2 n}, n \geq 2$, with $\theta \neq \mathrm{id}$, although it could still be the case that the open intervals are PL balls or spheres. This is false in general. For instance, recall Example 4.1.9, where we saw an interval in $\operatorname{Br}(\iota(\theta))$ which is not even graded.

Quasiparabolic $W$-sets, where $W$ is a Coxeter group, were introduced by Rains and Vazirani [36], and include all parabolic quotients $W^{J}, J \subseteq S$. We prove that Bruhat orders on quasiparabolic $W$-sets (under a boundedness assumption) form pircons. By Theorem 5.3.4, the open intervals are PL balls or spheres. This confirms a claim in [36]. In particular, it gives an alternative proof of the fact, known from Theorem 4.1.2, that the open intervals in $\operatorname{Br}\left(W^{J}\right)$ are PL balls or spheres. 



\section{Bibliography}

[1] N. Abdallah and A. Hultman, Combinatorial invariance of KazhdanLusztig-Vogan polynomials for fixed point free involutions, to appear in J. Algebraic Combin.

[2] A. Björner, Shellable and Cohen-Macaulay partially ordered sets, Trans. Amer. Math. Soc. 260 (1980), 159-183.

[3] A. Björner and F. Brenti, Combinatorics of Coxeter groups, Graduate Texts in Mathematics, vol. 231, Springer, New York, 2005.

[4] A. Björner and M. Wachs, Bruhat order of Coxeter groups and shellability, Adv. in Math. 43 (1982), 87-100.

[5] A. Björner and M. L. Wachs, Shellable nonpure complexes and posets. I, Trans. Amer. Math. Soc. 348 (1996), 1299-1327.

[6] F. Brenti, The intersection cohomology of Schubert varieties is a combinatorial invariant, European J. Combin. 25 (2004), 1151-1167.

[7] M. B. Can, Y. Cherniavsky, and T. Twelbeck, Lexicographic shellability of the Bruhat-Chevalley order on fixed-point-free involutions, Israel J. Math. 207 (2015), 281-299.

[8] C. Chevalley, Sur les décompositions cellulaires des espaces $G / B$, Algebraic groups and their generalizations: classical methods (University Park, PA, 1991), Proc. Sympos. Pure Math., vol. 56, Amer. Math. Soc., Providence, RI, 1994, pp. 1-23.

[9] G. Danaraj and V. Klee, Shellings of spheres and polytopes, Duke Math. J. 41 (1974), 443-451.

[10] V. V. Deodhar, Some characterizations of Bruhat ordering on a Coxeter group and determination of the relative Möbius function, Invent. Math. 39 (1977), 187-198. 
[11] (1982), 611-630.

[12] F. du Cloux, An abstract model for Bruhat intervals, European J. Combin. 21 (2000), 197-222.

[13] M. J. Dyer, Hecke algebras and reflections in Coxeter groups, Ph.D. thesis, University of Sydney, 1987.

[14] Hecke algebras and shellings of Bruhat intervals, Compositio Math. 89 (1993), 91-115.

[15] _ On minimal lengths of expressions of Coxeter group elements as products of reflections, Proc. Amer. Math. Soc. 129 (2001), 2591-2595.

[16] P. H. Edelman, The Bruhat order of the symmetric group is lexicographically shellable, Proc. Amer. Math. Soc. 82 (1981), 355-358.

[17] Z. Hamaker, E. Marberg, and B. Pawlowski, Involution words II: braid relations and atomic structures, J. Algebraic Combin. 45 (2017), 701743.

[18] J. Hu and J. Zhang, On involutions in symmetric groups and a conjecture of Lusztig, Adv. Math. 287 (2016), 1-30.

[19] _ On involutions in Weyl groups, J. Lie Theory 27 (2017), 671706.

[20] J. F. P. Hudson, Piecewise linear topology, University of Chicago Lecture Notes prepared with the assistance of J. L. Shaneson and J. Lees, W. A. Benjamin, Inc., New York-Amsterdam, 1969.

[21] A. Hultman, Fixed points of involutive automorphisms of the Bruhat order, Adv. Math. 195 (2005), 283-296.

[22] - The combinatorics of twisted involutions in Coxeter groups, Trans. Amer. Math. Soc. 359 (2007), 2787-2798.

[23] _ Fixed points of zircon automorphisms, Order 25 (2008), 85-90.

[24] (2008), 313-332.

[25] J. E. Humphreys, Reflection groups and Coxeter groups, Cambridge Studies in Advanced Mathematics, vol. 29, Cambridge University Press, Cambridge, 1990. 
[26] F. Incitti, The Bruhat order on the involutions of the hyperoctahedral group, European J. Combin. 24 (2003), 825-848.

[27] _ The Bruhat order on the involutions of the symmetric group, J. Algebraic Combin. 20 (2004), 243-261.

[28] _ Bruhat order on the involutions of classical Weyl groups, Adv. in Appl. Math. 37 (2006), 68-111.

[29] A. Mandel, Topology of oriented matroids, Ph.D. thesis, University of Waterloo, 1982.

[30] E. Marberg, Braid relations for involution words in affine Coxeter groups, preprint (2017), arXiv:1703.10437v3.

[31] M. Marietti, Algebraic and combinatorial properties of zircons, J. Algebraic Combin. 26 (2007), 363-382.

[32] J. Matoušek, Using the Borsuk-Ulam theorem, Universitext, SpringerVerlag, Berlin, 2003.

[33] H. Matsumoto, Générateurs et relations des groupes de Weyl généralisés, C. R. Acad. Sci. Paris 258 (1964), 3419-3422.

[34] B. Mühlherr, On isomorphisms between Coxeter groups, Des. Codes Cryptogr. 21 (2000), 189.

[35] R. A. Proctor, Classical Bruhat orders and lexicographic shellability, J. Algebra 77 (1982), 104-126.

[36] E. M. Rains and M. J. Vazirani, Deformations of permutation representations of Coxeter groups, J. Algebraic Combin. 37 (2013), 455-502.

[37] N. Reading, The cd-index of Bruhat intervals, Electron. J. Combin. 11 (2004), Research Paper 74, 25 pp.

[38] R. W. Richardson and T. A. Springer, The Bruhat order on symmetric varieties, Geom. Dedicata 35 (1990), 389-436.

[39] _ Complements to: The Bruhat order on symmetric varieties, Geom. Dedicata 49 (1994), 231-238.

[40] C. P. Rourke and B. J. Sanderson, Introduction to piecewise-linear topology, Springer-Verlag, New York-Heidelberg, 1972. 
[41] T. A. Springer, Some results on algebraic groups with involutions, Algebraic groups and related topics (Kyoto/Nagoya, 1983), Adv. Stud. Pure Math., vol. 6, North-Holland, Amsterdam, 1985, pp. 525-543.

[42] R. P. Stanley, Enumerative combinatorics. Vol. 1, Cambridge Studies in Advanced Mathematics, vol. 49, Cambridge University Press, Cambridge, 1997.

[43] J. Tits, Le problème des mots dans les groupes de Coxeter, Symposia Mathematica (INDAM, Rome, 1967/68), Vol. 1, Academic Press, London, 1969, pp. 175-185.

[44] M. L. Wachs, Poset topology: tools and applications, Geometric combinatorics, IAS/Park City Math. Ser., vol. 13, Amer. Math. Soc., Providence, RI, 2007, pp. 497-615. 


\section{Part II}

\section{Papers}





\section{Papers}

The papers associated with this thesis have been removed for copyright reasons. For more details about these see:

http://urn.kb.se/resolve?urn=urn:nbn:se:liu:diva-147976 